\title{
Synthesis of 6-(2-Furyl) and 6-(2-Thienyl)-4-trifluoromethylpyrimidinones and pyrimidines from 4-(2-Heteroaryl)-4-methoxy-1,1,1-trifluoro-3-buten-2-ones
}

\author{
Alex F. C. Flores, * Lucas Pizzuti, Sergio Brondani, Marcelo Rossato, Nilo Zanatta and \\ Marcos A. P. Martins
}

Departamento de Química, Universidade Federal de Santa Maria, 97105-900 Santa Maria-RS, Brazil

\begin{abstract}
Neste trabalho é apresentada a síntese, em rendimentos razoáveis (50-67\%), de novos sistemas biheterocíclicos, duas 6-(2-heteroaril)-4-trifluormetil-2-(1H)-pirimidinonas (2a,b) e uma série de dez 6-(2-heteroaril)-4-trifluormetilpirimidinas (3a,b - 7a,b) a partir da ciclocondensação de 1,1,1-trifluor-4-(2-heteroaril)-4-metoxi-3-buten-2-onas com uréia e amidinas. As estruturas de todos os compostos foram atribuídas pelos dados de análise elementar, espectrometria de massas e dados de $\mathrm{RMN}{ }^{1} \mathrm{H}$ e ${ }^{13} \mathrm{C}$. Os dados de $\mathrm{RMN}{ }^{1} \mathrm{H}$ e ${ }^{13} \mathrm{C}$ são mostrados de maneira sistemática. Também apresentamos os dados de difração de raios-X de um monocristal da 2-amino-6-(tien-2-il)-4-trifluormetilpirimidina (5b).
\end{abstract}

The synthesis of biheterocyclic systems 6-(2-furyl)-pyrimidines and 6-(2-thienyl)pyrimidines in reasonable yields (50-67\%), two 6-(2-heteroaryl)-4-trifluoromethyl-2-( $1 H$ )pyrimidinones $(\mathbf{2 a}, \mathbf{b})$ and a series of ten 6-(2-heteroaryl)-4-trifluoromethylpyrimidines (3a,b $7 \mathbf{a}, \mathbf{b})$ from the cyclocondensation of 1,1,1-trifluoro-4-(2-heteroaryl)-4-methoxy-3-buten-2-ones with urea and amidines is reported. Structures of all compounds have been elucidated by elemental analysis, mass spectrometry and ${ }^{1} \mathrm{H},{ }^{13} \mathrm{C}$ NMR measurements. The ${ }^{1} \mathrm{H}$ and ${ }^{13} \mathrm{C}$ NMR data are systematically reported. The X-ray diffraction data for monocrystal from 2-amino-4trifluoromethyl-6-(thien-2-yl)-pyrimidine (5b) are reported.

Keywords: 4-trifluoromethylpyrimidines, 1,1,1-trifluoro-4-methoxy-3-buten-2-ones, $[3+3]$ cyclocondensation

\section{Introduction}

Interest in perfluoroalkylated heterocyclic compounds is largely due to the fact that they have enhanced biological activity and can be used as medicinal or agricultural chemicals. ${ }^{1-8}$ Among them, fluorinated pyrimidines have been shown to possess high biological activities as bactericides, fungicides, analgesics, antipyretics and antiinflammatories. ${ }^{9-15}$

Perfluoroalkylated N-containing heterocycles can be obtained by various methods, including the widely used reactions of 1,3-dicarbonyl compounds with binucleophiles. For example, the cyclization of N-C-N blocks (urea, guanidines and amidines) with perfluoroalkyl containing 1,3-bielectrophiles (1,3-diketones, 1,3ketoesters, 1,3 -ketoamides, $\alpha, \beta$-enones) is considered the main procedure for the synthesis of perfluoroalkyl substituted pyrimidine derivatives. ${ }^{15-18}$

*e-mail: alexflores@smail.ufsm.br
Derivatives 2-substituted of the 6-(2-furyl)-4trifluoromethylpyrimidines and 6-(2-thienyl)-4trifluoromethylpyrimidines were early synthesized by cyclocondensation respectively of 1-(2-furyl)-4,4,4trifluorobutane-1,3-dione or 1-(2-thienyl)-4-trifluorobutane-1,3-dione with amidines $\mathrm{NH}_{2} \mathrm{C}(\mathrm{NH}) \mathrm{X}$ [ $\mathrm{X}=\mathrm{OR}$, $\left.\mathrm{NH}_{2}, \mathrm{SMe}\right]$, however only few analytical data were reported. ${ }^{19-23}$ The patent reports show only melting point as accessible analytical data for these compounds.

The 6-furyl and 6-thienyl derivatives of 2-amino-4trifluoromethylpyrimidine and 2-hydroxy (or 2-mercapto)4-trifluoromethylpyrimidine obtained from cyclocondensation of 1-(2-heteroaryl)-4,4,4-trifluorobutane-1,3-dione and guanidine or urea were applied in effective treatment of cardiorenal disease and in edema. ${ }^{19}$ Moreover had demonstrated eficient inhibitory activity in mevalonic acid incorporation during biosynthesis of cholesterol. ${ }^{20}$

Recently we have reported the synthesis of 4-(2-heteroaryl)4-methoxy-1,1,1-trifluoro-3-buten-2-ones as building blocks to construct promising trifluoromethyl containing 
biheterocyclic systems. ${ }^{9,24,25}$ The present work aimed to report efficient procedures for the systematic cyclocondensation of 4-(2-furyl)-4-methoxy-1,1,1-trifluoro-3-buten-2-one (1a) and 4-methoxy-4-(2-thienyl)-1,1,1-trifluoro-3-buten-2-one (1b) with urea, acetamidine, benzamidine, guanidine, 2-methyl-2pseudothiourea and $1 H$-pyrazole-1-carboxyamidine for synthesis of the series of biheterocyclic 6-(2-heteroaryl)-4trifluoromethylpyrimidinones 2 and 6-(2-heteroaryl)-4trifluoromethylpyrimidines 3-5, 7 and new three ring system 2-(pyrazol-1-yl)-4-trifluoromethyl-6-(2-heteroaryl)pyrimidines 6.

\section{Results and Discussion}

The 4-(2-heteroaryl)-4-methoxy-1,1,1-trifluoromethyl3-buten-2-ones $\mathbf{1 a}$ and $\mathbf{1 b}$ were prepared using the previously reported procedure. ${ }^{25}$ The 6-(2-furyl)-4trifluoromethyl-1H-pyrimidin-2-one $\mathbf{2 a}$ and the 6-(2thienyl)-4-trifluoromethyl-1H-pyrimidin-2-one $\mathbf{2 b}$ were prepared in very low yields $(<10 \%)$ from the reaction of 1a or $\mathbf{1 b}$ with urea in reflux EtOH or, even $i$-PrOH (Scheme 1). ${ }^{12}$ Several attempts to improve the yields by refluxing 1a or $\mathbf{1 b}$ with urea for long periods (2 days) without catalysis were unsuccessful. However, polymeric material was obtained in reactions in $\mathrm{MeOH}$ with Brönsted $\mathrm{HCl}$ catalysis at room temperature or with Lewis acid $\mathrm{BF}_{3} \cdot \mathrm{OEt}_{2}$ or $\mathrm{Ti}(\mathrm{O}-$ $i \mathrm{Pr})_{4}$ catalysis at reflux temperature $\left(>65^{\circ} \mathrm{C}\right)$ for long periods. ${ }^{15}$ Our experiments have demostrated that the best medium was anhydrous $i-\mathrm{PrOH}$ with drops of $\mathrm{BF}_{3} \cdot \mathrm{OEt}_{2}$ at $50{ }^{\circ} \mathrm{C}$ during $20 \mathrm{~h}$, furnishing reasonable yields (50\%) for $\mathbf{2 a}$ and $\mathbf{2 b} .{ }^{12}$ The ${ }^{1} \mathrm{H}$ NMR spectra have show a single set of signals (see, Experimental) indicating that compounds 2a and $\mathbf{2 b}$ exist as one of the possible tautomers. The 2(1H)pyrimidin-2-one structure was confirmed by characteristic signal from N-H at 12.93-12.95 ppm.

In the search for the optimum cyclocondensations condition for $\mathbf{1 a}, \mathbf{1 b}$ and amidines acetamidine hydrochloride was used as a model amidine. The cyclocondensations in $\mathrm{MeOH}$ or $i \mathrm{PrOH}$ under Brönsted $\mathrm{HCl}$ or Lewis $\mathrm{BF}_{3} \cdot \mathrm{OEt}_{2}$ catalysis were unsuccessful, the reactants were recovered. In contrast to the synthesis of 4-polyfluoroalkylpyrimidines by condensation in refluxing $i \mathrm{PrOH}$ under Lewis $\mathrm{BF}_{3} \cdot \mathrm{OEt}_{2}$ catalysis for 4 to $26 \mathrm{~h},{ }^{12}$ we have obtained products 6 -(2heteroaryl)-2-methyl-4-trifluoromethyl-pyrimidines $\mathbf{3 a}$ and $\mathbf{3 b}$ in good yields using alkaline medium, reacting acetamidine hydrochloride with a $1 \mathrm{~mol} \mathrm{~L}^{-1} \mathrm{KOH}$ aqueous solution and adding to $\mathrm{MeOH}$ solution of $\mathbf{1 a}$ or $\mathbf{1 b}$ at room temperature. TLC analyses during reaction period have revealed that the 1,3-dielectrophiles were consumed after 1 hour, furnishing good yields of the pyrimidines 3 . The 6-(2heteroaryl)-2-phenyl-4-trifluoromethyl-pyrimidines $\mathbf{4 a}, \mathbf{4 b}$, 2-amino-6-(2-heteroaryl)-4-trifluormethyl-pyrimidines $\mathbf{5 a}$, 5b, 6-(2-heteroaryl)-2-(1H-pyrazol-1-yl)-4-trifluoromethylpyrimidines $\mathbf{6 a}, \mathbf{6 b}$ and the 6-(2-heteroaryl)-2thiomethyl-4-trifluoromethyl-pyrimidines $7 \mathbf{a}, 7 \mathbf{b}$ were obtained in similar alkaline medium with the procedure described above. The structure of all compounds was determined from ${ }^{1} \mathrm{H},{ }^{13} \mathrm{C}$ and mass spectrometry. Based on our previous reports on the chemistry of 6-aryl-4trifluoromethyl-1H-pyrimidin-2-ones and 6-aryl-4trifluoromethyl-pyrimidines derived from 4-aryl-4-methoxy1,1,1-trifluoro-3-buten-2-ones, the assignment of each signal in the ${ }^{13} \mathrm{C}$ NMR spectra of compounds 2-7 was accurately established. ${ }^{15,16}$

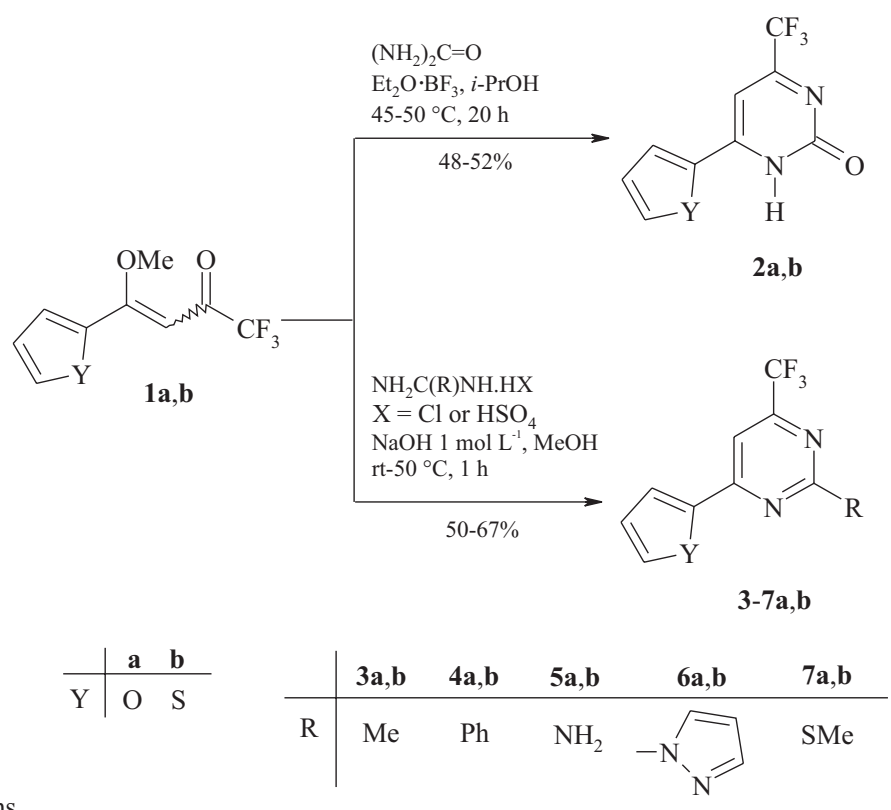

Scheme 1. $[3+3]$ cyclocondensations. 
Table 1. Crystal data ${ }^{\mathrm{a}}$ and structure refinement for 2-amino-4trifluoromethyl-6-(2-thienyl)-pirimidine (5b)

\begin{tabular}{|c|c|c|}
\hline \multicolumn{3}{|l|}{ Crystal data } \\
\hline Formula & \multicolumn{2}{|l|}{$\mathrm{C}_{9} \mathrm{H}_{6} \mathrm{~F}_{3} \mathrm{~N}_{3} \mathrm{~S}$} \\
\hline Habit & \multicolumn{2}{|l|}{ Colorless prisms } \\
\hline Size / $(\mathrm{mm})$ & \multicolumn{2}{|c|}{$0.240 \times 0.090 \times 0.079$} \\
\hline Symmetry & \multicolumn{2}{|l|}{ Monoclinic, $\mathrm{P} 2_{1 / \mathrm{c}}$} \\
\hline \multirow[t]{3}{*}{ Unit cell dimensions $/\left(\AA,^{\circ}\right)$} & $\mathrm{a}=5.0982(2)$ & $\alpha=90$ \\
\hline & $\mathrm{b}=19.4858(7)$ & $\beta=95.779(2)$ \\
\hline & $\mathrm{c}=10.1807(3)$ & $\gamma=90$ \\
\hline Volume / $\left(\AA^{3}\right), \mathrm{Z}$ & $1006.24(6), 4$ & \\
\hline$D_{\mathrm{c}} /\left(\mathrm{g} \mathrm{cm}^{-3}\right), F(000)$ & $1.619,496$ & \\
\hline$\mu /\left(\mathrm{mm}^{-1}\right)$ & 0.339 & \\
\hline$\theta$ range for data collection / $\left({ }^{\circ}\right)$ & 3.73 to 28.37 & \\
\hline Index ranges & $\begin{array}{l}-6 \leq h \leq 6, \\
-26 \leq k \leq 26 \\
-13 \leq 1 \leq 13\end{array}$ & \\
\hline Reflections collected & 11012 & \\
\hline Independent reflections $\left(\mathrm{R}_{-}{ }_{\text {in }}\right)$ & $2501(0.0426)$ & \\
\hline Completeness to $\theta$ & $99.4 \%$ & \\
\hline$T_{\min }-T_{\max }$ & $0.7957-1.0$ & \\
\hline Solution & Direct methods S & HELXS-97 \\
\hline Refinement method & Full matrix least- & quares on $F^{2}$ \\
\hline Data / restraints / parameters & $2501 / 0 / 146$ & \\
\hline Goodness-of-fit on $F^{2}$ & 0.947 & \\
\hline Final $R$ indices $[I>2 \sigma(I)]$ & $\mathrm{R} 1=0.0434, w \mathrm{R}$ & $=0.1209$ \\
\hline $\mathrm{R}$ indices, all data & $\mathrm{R} 1=0.0790, \mathrm{wR}$ & $=0.1389$ \\
\hline Largest diff. peak and hole / (e. $\left.\AA^{3}\right)$ & 0.202 and -0.282 & \\
\hline
\end{tabular}

The ${ }^{1} \mathrm{H}$ and ${ }^{13} \mathrm{C}$ spectra of products showed set of signals attributed to aromatic pyrimidines $\mathbf{3 a}, \mathbf{3 b}$ to $\mathbf{7 a}, \mathbf{7} \mathbf{b}$. The structure of compound 2-amino-6-(2-thienyl)-4trifluoromethyl-pyrimidine $\mathbf{5 b}$ was established by X-ray diffraction analysis. The overall view of the molecule is shown in Figure 1. The 6-(2-thienyl)-pyrimidine system is virtually coplanar (the mean deviation of the atoms from the plane is $0.008 \AA$ ). The thienyl ring suffers a rotational disorder, atoms $\mathrm{S} 1$ and $\mathrm{C} 7$ were modeled as exchanged with a minor occupancy fraction refined to $19.6 \%$. The

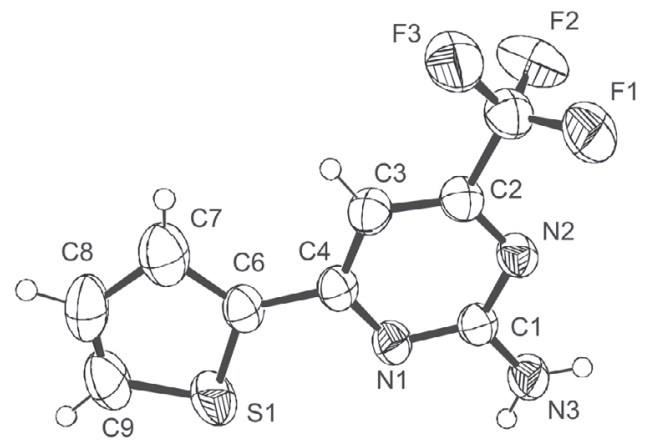

Figure 1. ORTEP Diagram of the X-ray crystal structure of $\mathbf{5 b}$ at a probability level of $50 \%$. trifluoromethyl group presents a rotational disorder; the three $\mathrm{F}$ atoms were refined in a position rotated approximately $45^{\circ}$ from their original positions at 5.3\% occupancy. Crystal data for $\mathbf{5 b}$ are shown in Table 1.

\section{Conclusions}

In conclusion, the biheterocyclic systems 6-(2-furyl)and 6-(2-thienyl)-pyrimidinones and 6-(2-furyl)- and 6-(2-thienyl)-pyrimidines were efficiently obtained by reacting 4-(2-heteroaryl)-4-methoxy-1,1,1-trifluoro-3buten-2-ones with $\mathrm{N}-\mathrm{C}-\mathrm{N}$ binucleophiles, in reasonable to good yields $(50-67 \%)$. The best reactional medium for urea cyclocondensation was under $\mathrm{BF}_{3} \cdot \mathrm{OEt}_{2}$ Lewis acid catalysis. However the cyclocondensations with hydrochoride amidines occurred only in alkaline media with $\mathrm{NaOH}, \mathrm{pH}>15$, furnishing good yields of 6-(2heteroaryl)-pyrimidines. The tricyclic systems 6-(2-furyl)and 6-(2-thienyl)-2-(1-pyrazolyl)-pyrimidines are new.

\section{Experimental}

The synthesis of 4-(2-heteroaryl)-4-methoxy-1,1,1trifluoro-3-buten-2-one, $\mathbf{1}$ has been reported elsewhere. ${ }^{20}$ Urea, amidines and $\mathrm{BF}_{3} \cdot \mathrm{OEt}_{2}$ were used as obtained from commercial suppliers. $\mathrm{MeOH}$ and $i$ - $\mathrm{PrOH}$ were purified before using. $1 \mathrm{~mol} \mathrm{~L}^{-1}$ solution of $\mathrm{NaOH}$ was prepared by the dissolution of $0.40 \mathrm{~g}$ of $\mathrm{NaOH}$ in $100 \mathrm{~mL}$ of distilled water. Yields listed in Table 2 are of isolated compounds. All melting points were determined on a Reichert Thermovar apparatus and are uncorrected. The ${ }^{1} \mathrm{H}$ and ${ }^{13} \mathrm{C}$ spectra were recorded at $298 \mathrm{~K}$ on a Bruker DPX 400 spectrometer $\left({ }^{1} \mathrm{H}\right.$ at $400.13 \mathrm{MHz},{ }^{13} \mathrm{C}$ at 100.63 $\mathrm{MHz}$ ) with digital resolution of $\pm 0.01 \mathrm{ppm}$. All the chemical shifts are expressed in ppm, ${ }^{1} \mathrm{H}$ and ${ }^{13} \mathrm{C}$ are reported with respect to internal TMS. $0.1 \mathrm{~mol} \mathrm{~L}^{-1} \mathrm{CDCl}_{3}$ solutions were used except with compounds $2,0.1 \mathrm{~mol}$ $\mathrm{L}^{-1}$ in DMSO- $d_{6}$. H-H and C-F coupling constants are in Hz. Mass spectra were registered in a HP 5973 MSD connected to a HP $6890 \mathrm{GC}$ and interfaced by a Pentium PC. The GC was equipped with a split-splitless injector, auto-sampler, cross-linked HP-5 capillary column (30 $\mathrm{m}, 0.32 \mathrm{~mm}$ of internal diameter), and helium was used as the carrier. Elemental analyses were performed on a Perkin-Elmer 2400 CHN elemental analyzer (São Paulo University-São Paulo, Brazil). The crystal and molecular structure of $\mathbf{5 b}$ was determined by a single crystal X-ray diffraction study. Data were recorded on a Bruker Kappa Apex II CCD area detector with graphite monochromatized Mo Ka radiation (1 $0.71073 \AA$ ). The data were processed with SAINT and SADABS. The 
structure was solved by direct methods (SHELXS-97) ${ }^{26}$ and additional atoms were located in the difference Fourier map and refined on $\mathrm{F}^{2}$ using the SHELXTL and Wingx packages. ${ }^{27}$

6-(2-Furyl) [(2-thienyl)]-4-trifluoromethylpyrimidin-2-one $(2 \boldsymbol{a}, \boldsymbol{b})$. General Procedure

Compounds 1a ( $3 \mathrm{mmol}, 0.67 \mathrm{~g})$ or $\mathbf{1 b}(3 \mathrm{mmol}, 0.71$ g) and urea $(3.3 \mathrm{mmol}, 0.21 \mathrm{~g})$ were dissolved in $3 \mathrm{~mL}$ of anhydrous $i$-PrOH. Then, 3 drops of $\mathrm{BF}_{3} \cdot \mathrm{Et}_{2} \mathrm{O}$ were added and the mixture was stirred at $50^{\circ} \mathrm{C}$ for $20 \mathrm{~h}$. The solvent was partially evaporated and the product crystallized by cooling. The solid was filtered, washed with cold water and recrystallized from $\mathrm{MeOH}$ to give $\mathbf{2 a}$ and $\mathbf{2 b}$, respectively.

\section{6-(2-Furyl)-4-trifluoromethyl-1H-pyrimidin-2-one, $2 a$}

Yield $48 \%$; mp 282-284 ${ }^{\circ} \mathrm{C}$; ${ }^{1} \mathrm{H}$ NMR (400 MHz, DMSO- $d_{6}$ ): $\delta 6.82(\mathrm{dd}, 1 \mathrm{H}, J 3.5, J 1.66, \mathrm{H} 4$ '), 7.48 (s, $1 \mathrm{H}, \mathrm{H} 5), 7.67$ (d, 1H, J 3.5, H3'), 8.07 (d, 1H, $J$ 1.66, H5'), 12.93 (s, 1H, NH); ${ }^{13} \mathrm{C}$ NMR (100 MHz, DMSO$\left.d_{6}\right): \delta 163.1,157.9\left({ }^{2} J_{\mathrm{CF}} 35\right), 157.0,148.5,147.6,120.2$ $\left(J_{\mathrm{CF}} 277\right), 115.8,112.9,100.6$; MS $(70 \mathrm{eV}) \mathrm{m} / z 230\left(\mathrm{M}^{+}\right.$, 100), 118 (16), 90 (11), 63 (18). Anal. Calc. for $\mathrm{C}_{9} \mathrm{H}_{5} \mathrm{~F}_{3} \mathrm{~N}_{2} \mathrm{O}_{2}: \mathrm{C}, 46.97 ; \mathrm{H}, 2.19 ; \mathrm{N}, 12.17$. Found: C, 46.87; H, 2.18; N, 12.05 .

6-(2-Thienyl)-4-trifluoromethyl-1H-pyrimidin-2-one, $2 \boldsymbol{b}$

Yield $52 \%$; mp 266-268 ${ }^{\circ} \mathrm{C}$; ${ }^{1} \mathrm{H}$ NMR $(400 \mathrm{MHz}$, DMSO- $\left.d_{6}\right): 7.24$ (dd, $1 \mathrm{H}, J 4.8, J 3.4, \mathrm{H}^{\prime}$ ) $; 7.89$ (d, $1 \mathrm{H}$, $J$ 4.8, H3'); 7.95 (s, 1H, H5); 8.24 (d, 1H, J 3.4, H5'); 12.95 (s, 1H, NH); ${ }^{13} \mathrm{C}$ NMR (100 MHz, DMSO-d6): $\delta 165.1,163.5,156.6\left({ }^{2} J_{\mathrm{CF}} 35\right), 140.4,132.6,130.9,128.7$, $120.2\left(J_{\text {CF }} 274\right), 103.5$; MS $(70 \mathrm{eV}) \mathrm{m} / z 246\left(\mathrm{M}^{+}, 100\right)$, 227 (10), 134 (30), 69 (17). Anal. Calc. for $\mathrm{C}_{-} \mathrm{H}_{5} \mathrm{~F}_{3} \mathrm{~N}_{2} \mathrm{SO}$ : C, 43.91; H, 2.05; N, 11.38. Found: C, 43.59; H, 2.02; N, 11.23 .

6-(2-Furyl) [(2-thienyl)]-4-trifluoromethylpyrimidines (3$7 \boldsymbol{a}, \boldsymbol{b})$. General Procedure

Acetamidine hydrochloride ( $3 \mathrm{mmol})$ was added to an aqueous $\mathrm{KOH} 1 \mathrm{~mol} \mathrm{~L}^{-1}$ solution $(3 \mathrm{~mL})$. The resulting solution was added to a $\mathrm{MeOH}$ solution of compounds 1a (3 mmol, $0.66 \mathrm{~g})$ or $1 \mathbf{b}(3 \mathrm{mmol}, 0.71 \mathrm{~g})$. The resulting mixture was stirred at room temperature at $50{ }^{\circ} \mathrm{C}$ for 1 h. Then $\mathrm{MeOH}$ was evaporated and the resulting material was diluted with $\mathrm{CHCl}_{3}(50 \mathrm{~mL})$ and washed with water $(3 \times 20 \mathrm{~mL})$. The organic solution was dried over anhydrous $\mathrm{MgSO}_{4}$ and the solvent was removed, the solid products were recrystallized from hexane, furnishing the pure crystalline products $\mathbf{3 a}, \mathbf{3 b}, \mathbf{5 a}, \mathbf{5 b}, \mathbf{6} \mathbf{a}$ and $\mathbf{6 d}$. The compounds $7 \mathbf{a}$ and $\mathbf{7 b}$ were oils purified by chromatography column with hexane:chloroform $(1: 1)$ as eluent. When the precipitated product was formed, it was filtered, washed with water and dried over $\mathrm{CaCl}_{2}$. The products $\mathbf{4 a}, \mathbf{4} \mathbf{b}$ were obtained pure (GC-MS, ${ }^{1} \mathrm{H}$ NMR).

\section{6-(2-Furyl)-2-methyl-4-trifluoromethylpyrimidine, $3 \boldsymbol{a}$}

Yield $48 \%$ (71\%, ref. 28); mp $41-43{ }^{\circ} \mathrm{C}$; ${ }^{1} \mathrm{H}$ NMR (400 MHz, $\left.\mathrm{CDCl}_{3}\right): 2.82$ (s, 3H, Me); 6.62 (dd, $1 \mathrm{H}, J$ $3.52 \mathrm{~Hz}, J 1.76 \mathrm{~Hz}, \mathrm{H} 4$ '); 7.38 (d, 1H, J $3.52 \mathrm{~Hz}, \mathrm{H} 3$ '); 7.65 (d, 1H, $J 1.76 \mathrm{~Hz}, \mathrm{H} 5$ '); 7.72 (s, H5); ${ }^{13} \mathrm{C}$ NMR $\left(100 \mathrm{MHz}, \mathrm{CDCl}_{3}\right): \delta 169.4,157.4,156.1\left({ }^{2} J_{\mathrm{CF}} 35 \mathrm{~Hz}\right)$, $151.0,145.8,120.2\left(J_{\mathrm{CF}} 274\right), 113.9,112.8,107.5\left({ }^{4} J_{\mathrm{CF}}\right.$ $2.8 \mathrm{~Hz}), 25.9$; (MS (70 eV) m/z $228\left(\mathrm{M}^{+}, 95\right), 209$ (27), 118 (100), 90 (48), 63 (78). Anal. Calc. for $\mathrm{C}_{10} \mathrm{H}_{7} \mathrm{~F}_{3} \mathrm{~N}_{2} \mathrm{O}$ : C, 52.64; H, 3.09; N, 12.28. Found: C, 52.82; H, 3.36; N, 12.38 .

\section{2-Methyl-6-(2-thienyl)-4-trifluoromethylpyrimidine, $3 \boldsymbol{b}$}

Yield $53 \%$; mp $47-49{ }^{\circ} \mathrm{C}$; ${ }^{1} \mathrm{H}$ NMR $(400 \mathrm{MHz}$, $\left.\mathrm{CDCl}_{3}\right): 2.81$ (s, 3H, Me); 7.18 (dd, 1H, J 5.01, J 3.79 Hz, H4'); 7.59 (dd, 1H, J $5.01 \mathrm{~Hz}, J 1.04 \mathrm{~Hz}, \mathrm{H} 3$ '); 7.65 (s,1H, H5); $7.84\left(\mathrm{dd}, 1 \mathrm{H}, J 3.79 \mathrm{~Hz}, J 1.04 \mathrm{~Hz}, \mathrm{H} 5\right.$ '); ${ }^{13} \mathrm{C}$ NMR (100 MHz, $\left.\mathrm{CDCl}_{3}\right): \delta 169.6,161.1,155.9\left({ }^{2} J_{\mathrm{CF}} 35\right.$ $\mathrm{Hz}), 141.3,131.5,128.7,128.6,120.2\left(J_{\mathrm{CF}} 273 \mathrm{~Hz}\right), 107.8$ $\left({ }^{4} J_{\mathrm{CF}} 2.8 \mathrm{~Hz}\right), 25.9$; MS $(70 \mathrm{eV}) \mathrm{m} / z 244\left(\mathrm{M}^{+}, 100\right), 225$ (13), 134 (72), 69 (21). Anal. Calc. for $\mathrm{C}_{10} \mathrm{H}_{7} \mathrm{~F}_{3} \mathrm{~N}_{2} \mathrm{~S}$ : C, 49.18; H, 2.89; N, 11.47. Found: C, 48.78; H, 2.91; N, 11.41 .

\section{6-(2-Furyl)-2-phenyl-4-trifluoromethylpyrimidine, $4 a$}

Yield $61 \%$ (69\%, ref. 29); mp 66-68 ${ }^{\circ} \mathrm{C} ;{ }^{1} \mathrm{H}$ NMR (400 MHz, $\left.\mathrm{CDCl}_{3}\right)$ : 7.46-7.51 (m, 3H, Ph); 7.47 (d, 1H, $J 3.3 \mathrm{~Hz}, \mathrm{H} 3$ '); 7.57 (dd, 1H, J $3.3 \mathrm{~Hz}, J 1.7 \mathrm{~Hz}, \mathrm{H} 4$ '); 7.63 (d, 1H, J $1.7 \mathrm{~Hz}, \mathrm{H} 5$ '); 7.74 (s, 1H, H5); 8.52$8.54(\mathrm{~m}, 2 \mathrm{H}, \mathrm{Ph}) ;{ }^{13} \mathrm{C} \mathrm{NMR}\left(100 \mathrm{MHz}, \mathrm{CDCl}_{3}\right): \delta 165.2$, 157.6, $156.6\left({ }^{2} J_{\mathrm{CF}} 36 \mathrm{~Hz}\right), 151.4,145.8,136.1,131.5$, 128.6, 128.5, $120.8\left(J_{\mathrm{CF}} 274 \mathrm{~Hz}\right), 113.8,112.8,107.9$ $\left({ }^{4} J_{\mathrm{CF}} 2.7 \mathrm{~Hz}\right) ; \mathrm{MS}(70 \mathrm{eV}) \mathrm{m} / z 290\left(\mathrm{M}^{+}, 96\right), 271(16)$, 118 (100), 90 (52), 63 (75). Anal. Calc. for $\mathrm{C}_{15} \mathrm{H}_{9} \mathrm{~F}_{3} \mathrm{~N}_{2} \mathrm{O}$ : C, 62.07; H, 3.13; N, 9.65. Found: C, 61.54; H, 3.10; N, 9.38 .

\section{2-Phenyl-6-(2-thienyl)-4-trifluoromethylpyrimidine, $4 \boldsymbol{b}$}

Yield $67 \%$ (67\%, ref. 28); mp 104-106 ${ }^{\circ} \mathrm{C}$; ${ }^{1} \mathrm{H}$ NMR (400 MHz, $\mathrm{CDCl}_{3}$ ): 7.17 (dd, 1H, J 5.0, J 3.8 Hz, H4'); 7.48-7.51 (m, $3 \mathrm{H}, \mathrm{Ph}) ; 7.57$ (dd, $1 \mathrm{H}, J 5.0 \mathrm{~Hz}, J 1.05 \mathrm{~Hz}$, H3'); 7.65 (s, 1H, H5); 7.85 (dd, 1H, J $3.8 \mathrm{~Hz}, J 1.05 \mathrm{~Hz}$, 
$\mathrm{H}^{\prime}$ ); 8.53 - 8.56 (m, 2H, Ph); ${ }^{13} \mathrm{C} \mathrm{NMR}\left(100 \mathrm{MHz}, \mathrm{CDCl}_{3}\right)$ : $\delta 165.3,161.2,156.4\left({ }^{2} J_{\mathrm{CF}} 36 \mathrm{~Hz}\right), 141.8,136.1,131.5$, $131.4,128.7,128.6,128.5,120.8\left(J_{\mathrm{CF}} 274 \mathrm{~Hz}\right), 108.2\left({ }^{4} J_{\mathrm{CF}}\right.$ $2.8 \mathrm{~Hz})$; MS (70 eV) m/z $306\left(\mathrm{M}^{+}, 100\right), 287$ (5), 134 (73), 69 (8). Anal. Calc. for $\mathrm{C}_{15} \mathrm{H}_{9} \mathrm{~F}_{3} \mathrm{~N}_{2} \mathrm{~S}: \mathrm{C}, 58.82 ; \mathrm{H}, 2.96$; N, 9.15. Found: C, 58.80; H, 3.12; N, 8.72.

\section{2-Amino-6-(2-furyl)-4-trifluoromethylpyrimidine, $5 \boldsymbol{a}$}

Yield $50 \%$; mp 130-132 ${ }^{\circ} \mathrm{C}$; ${ }^{1} \mathrm{H}$ NMR (400 MHz, $\left.\mathrm{CDCl}_{3}\right): 5.61\left(\mathrm{~s}, 2 \mathrm{H}, \mathrm{NH}_{2}\right) ; 6.58(\mathrm{dd}, 1 \mathrm{H}, J 3.52 \mathrm{~Hz}, J$ $1.73 \mathrm{~Hz}, \mathrm{H} 4$ '); 7.23 (dd, 1H, J 3.52 Hz, J 0.7 Hz, H3'); 7.24 (s, 1H, H5); 7.61 (dd, 1H, J 1.73 Hz, J 0.7 Hz, H5'); ${ }^{13} \mathrm{C} \mathrm{NMR}\left(100 \mathrm{MHz}, \mathrm{CDCl}_{3}\right): \delta 163.2,158.7,157.2\left({ }^{2} J_{\mathrm{CF}}\right.$ $35 \mathrm{~Hz}), 151.1,145.6,120.6\left(J_{\mathrm{CF}} 274 \mathrm{~Hz}\right), 113.8,112.8$, $101.2\left({ }^{4} J_{\mathrm{CF}} 2.9 \mathrm{~Hz}\right)$; MS (70 eV) $\mathrm{m} / z 229\left(\mathrm{M}^{+}, 100\right), 118$ (35), 90 (24), 63 (37). Anal. Calc. for $\mathrm{C}_{9} \mathrm{H}_{6} \mathrm{~F}_{3} \mathrm{~N}_{3} \mathrm{O}: \mathrm{C}, 47.17$; H, 2.64; N, 18.34. Found: C, 48.00; H, 3.20; N, 18.38.

\section{2-Amino-6-(2-thienyl)-4-trifluoromethylpyrimidine, $\mathbf{5 b}$}

Yield $51 \%$; mp 141-143 ${ }^{\circ} \mathrm{C}$; ${ }^{1} \mathrm{H}$ NMR (400 MHz, $\left.\mathrm{CDCl}_{3}\right): 5.57$ (s, 2H, $\left.\mathrm{NH}_{2}\right) ; 7.15(\mathrm{dd}, 1 \mathrm{H}, J 5.0 \mathrm{~Hz}, J 3.8$ Hz, H4'); 7.19 (s, 1H, H5); 7.54 (dd, 1H, J 5.0 Hz, J 1.04 Hz, H3'); 7.77 (dd, 1H, J $3.8 \mathrm{~Hz}, J 1.04 \mathrm{~Hz}, \mathrm{H} 5$ '); ${ }^{13} \mathrm{C}$ NMR (100 MHz, $\left.\mathrm{CDCl}_{3}\right): \delta 163.1,162.4,157.0\left({ }^{2} J_{\mathrm{CF}} 35\right.$ $\mathrm{Hz}), 141.5,130.9,128.6,128.4,120.6\left(J_{\mathrm{CF}} 274 \mathrm{~Hz}\right), 101.4$ $\left({ }^{4} J_{\mathrm{CF}} 2.9 \mathrm{~Hz}\right) ; \mathrm{MS}(70 \mathrm{eV}) \mathrm{m} / z 245\left(\mathrm{M}^{+}, 100\right), 226(10)$, 204 (45), 134 (59), 69 (28); Anal. Calc. for $\mathrm{C}_{9} \mathrm{H}_{6} \mathrm{~F}_{3} \mathrm{~N}_{3} \mathrm{~S}$ : C, 44.08; H, 2.47; N, 17.14. Found: C, 44.70; H, 2.65; N, 16.59 .

6-(2-Furyl)-2-(1H-pyrazol-1-yl)-4-trifluoromethylpyrimidine, $\mathbf{6 a}$

Yield $54 \%$; mp 108-110 ${ }^{\circ} \mathrm{C} ;{ }^{1} \mathrm{H}$ NMR (400 MHz, $\mathrm{CDCl}_{3}$ ): 6.53 (dd, 1H, J 2.8 Hz, J $1.6 \mathrm{~Hz}, \mathrm{H} 4-\mathrm{pyr}$ ); 6.64 (dd, 1H, J 3.4 Hz, J 1.8 Hz, H4'); 7.57 (d, 1H, J 3.4 Hz, H3'); 7.69 (d, 1H, J 1.6 Hz, H5-pyr); 7.76 (s, 1H, H5); 7.89 (d, 1H, J 1.8 Hz, H5'); 8.65 (d, 1H, J 2.8 Hz, H3pyr); ${ }^{13} \mathrm{C}$ NMR $\left(100 \mathrm{MHz}, \mathrm{CDCl}_{3}\right): \delta 159.4,157.2\left({ }^{2} J_{\mathrm{CF}}\right.$ $37 \mathrm{~Hz}), 156.3,150.4,146.7,144.4,129.8,120.2\left(J_{\mathrm{CF}} 274\right.$ $\mathrm{Hz}), 115.6,113.2$, 109.8, 107.6; MS (70 eV) m/z $280\left(\mathrm{M}^{+}\right.$, 100), 261 (8), 213 (75), 118 (8), 90 (8), 63 (14). Anal. Calc. for $\mathrm{C}_{12} \mathrm{H}_{7} \mathrm{~F}_{3} \mathrm{~N}_{4} \mathrm{O}: \mathrm{C}, 51.44 ; \mathrm{H}, 2.52 ; \mathrm{N}, 19.99$. Found: C, 50.84; H, 2.64; N, 20.09.

\section{2-(1H-Pyrazol-1-yl)-6-(2-thienyl) 4-trifluoromethyl-} pyrimidine, $\mathbf{6} \boldsymbol{b}$

Yield $58 \%$; mp 116-118 ${ }^{\circ} \mathrm{C}$; ${ }^{1} \mathrm{H}$ NMR (400 MHz, $\mathrm{CDCl}_{3}$ ): 6.53 (dd, 1H, J 2.8 Hz, J $\left.1.58 \mathrm{~Hz}, \mathrm{H} 4-\mathrm{pyr}\right) ; 7.21$ (dd, 1H, J 4.99 Hz, J 3.83 Hz, H4'); 7.65 (dd, 1H, J 4.99 Hz, J 1.04 Hz, H3'); 7.68 (s, 1H, H5); 7.89 (d, 1H, J 1.58 Hz, H3-pyr); 7.95 (dd, 1H, J 3.83 Hz, J 1.04 Hz, H5');
8.66 (d, 1H, J $2.7 \mathrm{~Hz}, \mathrm{H} 5-\mathrm{pyr}) ;{ }^{13} \mathrm{C}$ NMR (100 MHz, $\left.\mathrm{CDCl}_{3}\right): \delta 163.2,157.7\left({ }^{2} J_{\mathrm{CF}} 36 \mathrm{~Hz}\right), 156.2,144.5,140.3$, 132.5, 129.9, 129.8, 128.8, $120.2\left(J_{\mathrm{CF}} 274 \mathrm{~Hz}\right), 109.1$, 107.9; MS (70 eV) m/z $296\left(\mathrm{M}^{+}, 100\right), 287$ (8), 229 (82), 134 (15), 69 (9). Anal. Calc. for $\mathrm{C}_{12} \mathrm{H}_{7} \mathrm{~F}_{3} \mathrm{~N}_{4} \mathrm{~S}$ : C, 48.65; $\mathrm{H}$, 2.38; N, 18.91. Found: C, 48.76; H, 2.47; N, 18.90.

6-(2-Furyl)-2-tiomethyl-4-trifluoromethylpyrimidine, 7a

Yield $52 \%$, oil; ${ }^{1} \mathrm{H}$ NMR (400 $\mathrm{MHz}, \mathrm{CDCl}_{3}$ ): 2.61 (s, 3H, SMe); 6.59 (dd, 1H, J 3.52 Hz, J 1.71 Hz, H4'); 7.36 (dd, 1H, J $3.52 \mathrm{~Hz}, J 0.71 \mathrm{~Hz}, \mathrm{H} 3$ '); 7.51 (s, 1H, H5); 7.63 (dd, 1H, J $\left.1.71 \mathrm{~Hz}, J 0.71 \mathrm{~Hz}, \mathrm{H} 5{ }^{\prime}\right) ;{ }^{13} \mathrm{C} \mathrm{NMR}$ $\left(100 \mathrm{MHz}, \mathrm{CDCl}_{3}\right): \delta 174.0,157.1,156.0\left({ }^{2} J_{\mathrm{CF}} 36 \mathrm{~Hz}\right)$, $150.7,146.1,120.4\left(J_{\mathrm{CF}} 274 \mathrm{~Hz}\right), 114.2,112.8,105.3$, 13.8; MS (70 eV) m/z $260\left(\mathrm{M}^{+}, 100\right), 241$ (12), 214 (38), 118 (18), 90 (13), 63 (28). Anal. Calc. for $\mathrm{C}_{10} \mathrm{H}_{7} \mathrm{~F}_{3} \mathrm{~N}_{2} \mathrm{OS}$ : C, 46.15; H, 2.71; N, 10.76. Found: C, 46.10; H, 2.80; $\mathrm{N}, 10.78$.

6-(2-Thienyl)-2-tiomethyl-4-trifluoromethylpyrimidine, $7 \boldsymbol{b}$

Yield $53 \%$, oil; ${ }^{1} \mathrm{H}$ NMR (400 MHz, $\left.\mathrm{CDCl}_{3}\right): 2.61$ (s, 3H, SMe); 7.15 (dd, 1H, J 5.0 Hz, J 3.9 Hz, H4'); 7.43 (s, 1H, H5); 7.56 (dd, 1H, J 5.0 Hz, J 1.1 Hz, H3'); 7.80 (dd, $\left.1 \mathrm{H}, J 3.90 \mathrm{~Hz}, J 1.10 \mathrm{~Hz}, \mathrm{H} 5{ }^{\prime}\right) ;{ }^{13} \mathrm{C} \mathrm{NMR}(100 \mathrm{MHz}$, $\left.\mathrm{CDCl}_{3}\right): \delta 174.2,162.7,155.8\left({ }^{2} J_{\mathrm{CF}} 36 \mathrm{~Hz}\right), 140.9,131.7$, 129.1, 128.6, $120.4\left(J_{\mathrm{CF}} 274 \mathrm{~Hz}\right), 105.7,14.1$; MS (70 eV) $m / z 276$ (M+100), 257 (14), 229 (60), 134 (45), 69 (16). Anal. Calc. for $\mathrm{C}_{10} \mathrm{H}_{7} \mathrm{~F}_{3} \mathrm{~N}_{2} \mathrm{~S}_{2}$ : C, 43.47; H, 2.55; N, 10.14. Found: C, 43.55; H, 2.60; N, 10.20.

Table 2. Bond length $(\AA)$ and angles $\left(^{\circ}\right)$ for 2-amino-4-trifluoromethyl6-(2-thienyl)-pyrimidine (5b)

\begin{tabular}{llll}
\hline Bond & Length $/(\AA)$ & Bonds & Angle / $\left(^{\circ}\right)$ \\
\hline C1-N3 & $1.338(2)$ & N3-C1-N1 & $117.62(15)$ \\
C1-N1 & $1.346(2)$ & N3-C1-N2 & $116.93(15)$ \\
C1-N2 & $1.348(2)$ & N1-C1-N2 & $125.45(16)$ \\
C2-N2 & $1.331(2)$ & N2-C2-C3 & $124.68(16)$ \\
C2-C3 & $1.365(3)$ & N2-C2-C10 & $113.89(16)$ \\
C2-C10 & $1.508(3)$ & C3-C2-C10 & $121.40(17)$ \\
C3-C4 & $1.404(3)$ & C2-C3-C4 & $115.87(17)$ \\
C3-H3 & 0.9300 & C2-C3-H3 & 122.1 \\
C4-N1 & $1.328(2)$ & C4-C3-H3 & 122.1 \\
C4-C6 & $1.460(2)$ & N1-C4-C3 & $121.50(16)$ \\
C6-C7 & $1.511(3)$ & N1-C4-C6 & $116.86(16)$ \\
C6-S1 & $1.681(2)$ & C3-C4-C6 & $121.65(17)$ \\
C7-C8 & $1.476(3)$ & C4-C6-C7 & $126.76(16)$ \\
C7-H7 & 0.930 & C4-C6-S1 & $119.59(15)$ \\
C8-C9 & $1.330(4)$ & C7-C6-S1 & $113.64(12)$ \\
C8-H8 & 0.930 & C8-C7-C6 & $102.90(17)$ \\
C9-S1 & $1.655(3)$ & C8-C7-H7 & 128.5 \\
C9-H9 & 0.930 & C6-C7-H7 & 128.5 \\
C10-F3 & $1.324(2)$ & C9-C8-C7 & $116.2(2)$ \\
C10-F2 & $1.326(2)$ & C9-C8-H8 & 121.9 \\
C10-F1 & $1.328(2)$ & C7-C8-H8 & 121.9 \\
N3-H3a & 0.860 & C8-C9-H9 & $114.51(18)$ \\
N3-H3b & 0.860 & S1-C9-H9 & 122.7 \\
\hline
\end{tabular}




\section{Acknowledgments}

Financial support from Conselho Nacional de Desenvolvimento Científico e Tecnológico ( $\mathrm{CNPq})$ and Fundação de Amparo à Pesquisa do Estado do Rio Grande do Sul (FAPERGS) is acknowledged. The Bruker X-ray diffractometer was funded by infrastructure grant from the Financiadora de Estudos e Projetos (CT-INFRA/ FINEP). Both of us L.Pizzuti and M.Rossato thank CNPq for fellowships.

\section{Supplementary Information}

Supplementary data ${ }^{1} \mathrm{H},{ }^{13} \mathrm{C}$ NMR and mass spectra are available free of charge at http://jbcs.sbq.org.br, as PDF file.

\section{References}

1. Welch, J. T.; Tetrahedron 1987, 43, 3123.

2. Filler, R.; Kobayashi, Y.; Yagupolskii, L. M.; Organofluorine Compounds in Medical Applications, Elsevier: Amsterdam, 1993.

3. McClinton, M. A.; McClinton, D. A.; Tetrahedron 1992, 48, 6555.

4. Tice, C. M.; Bryman, L. M.; Tetrahedron 2001, 57, 2689.

5. Burger, K.; Wacherpfenninh, U.; Brunner, E.; Adv. Heterocycl. Chem. 1995, 60, 1.

6. Singh, S. P.; Sehgal, S.; Singh, L.; Dhawan, S. N.; Indian J. Chem. 1990, 29B, 310.

7. Yamaguchi, Y.; Katsuyama, I.; Funabiki, K.; Matsui, M.; Shibata, K.; J. Heterocycl. Chem. 1998, 35, 805.

8. Shavnya, A.; Sakya, S. M.; Minich, M. L.; Rast, B.; DeMello, K. L.; Jaynes, B. H.; Tetrahedron Lett. 2005, 46, 6887.

9. Martins, M. A. P.; Pereira, C. M. P.; Zimmermann, N. E. K.; Cunico, W.; Moura, S.; Beck, P.; Zanatta, N.; Bonacorso, H. G.; J. Fluorine Chem. 2003, 123, 261.

10. Jones, B. G.; Branch, S. K.; Thompson, A. S.; Threadgill, M. D.; J. Chem. Soc., Perkin Trans. 1 1996, 2685.
11. Sloop, J. C.; Bumgardner, C. L.; Loehle, W. D.; J. Fluorine Chem. 2002, 118, 135.

12. Pashkevich, K. I; Sevenard, D. V.; Khomutov, O. G.; Vorontsov, I. I.; Russ. Chem. Bull., Int. Ed. 2001, 50, 669.

13. Habeeb, A. G.; Praveen Rao, P. N.; Knaus, E. E.; J. Med. Chem. 2001, 44, 3039.

14. Clark, R. D.; J. Agric. Food Chem. 1996, 44, 3643.

15. Bonacorso, H. G.; Lopes, I. S.; Wastowski, A. D.; Zanatta, N.; Martins, M. A. P. ; J. Fluorine Chem. 2003, 120, 29.

16. Burgart, Y. V.; Kuzueva, O. G.; Pryadeina, M. V. ; Kappe, C. O.; Saloutin, V. I.; Russ. J. Org. Chem. 2001, 37, 869.

17. Zanatta, N.; Flores, D. C.; Madruga, C. C.; Faoro, D.; Flores, A. F. C.; Bonacorso, H. G.; Martins, M. A. P.; Synthesis 2003, 894.

18. Sevenard, D. V.; Khomutov, O. G.; Koryakova, O. V.; Sattarova, V. V.; Kodess, M. I.; Stelten, J.; Loop, I.; Lork, E.; Pashkevich, K. I. ; Röschenthaler, G.-V.; Synthesis 2000, 1738.

19. Rorig, K. J.; US pat. 2748119 1956. (CA 51:5886)

20. Rorig, K. J.; Wagner, H. A.; US pat. 3149109 1964. (CA 63:39159)

21. Kucerovy, A.; Mattner, P. G.; Hathaway, J. S.; Repic, O.; Synth. Commun. 1990, 20, 913.

22. Kreutzberger, A.; Tesch, U. H.; Arzneim. Forsch. 1978, $28,235$.

23. Dilli, S.; Robards, K.; Austr. J. Chem. 1978, 31, 1833.

24. Bonacorso, H. G.; Martins, M. A. P.; Bittencourt, S. R. T.; Lourega, R. V.; Zanatta, N.; Flores, A. F. C.; J. Fluorine Chem. 1999, 99, 177.

25. Flores, A. F. C.; Brondani, S.; Zanatta, N.; Rosa, A.; Martins, M. A. P.; Tetrahedron Lett. 2002, 43, 8701.

26. Sheldrick, G. M.; SHELXS-97, Göttingen: Germany, 1997.

27. Farrugia, L. J.; J. Appl. Crystallogr. 1999, 32, 837.

28. Funabiki, K.; Nakamura, H.; Matsui, M.; Shibata, K.; Synlett 1999, 756.

29. Yu, H.-B.; Huang, W.-Y; J. Fluorine Chem. 1997, 84, 65.

Received: February 6, 2007 Web Release Date: October 19, 2007 


\section{Synthesis of 6-(2-Furyl) and 6-(2-Thienyl)-4-trifluoromethylpyrimidinones and -pyrimidines from 4-(2-Heteroaryl)-4-methoxy-1,1,1-trifluoro-3-buten-2-ones}

Alex F. C. Flores, " Lucas Pizzuti, Sergio Brondani, Marcelo Rossato, Nilo Zanatta and Marcos A. P. Martins

Departamento de Química, Universidade Federal de Santa Maria, 97105-900 Santa Maria-RS, Brazil

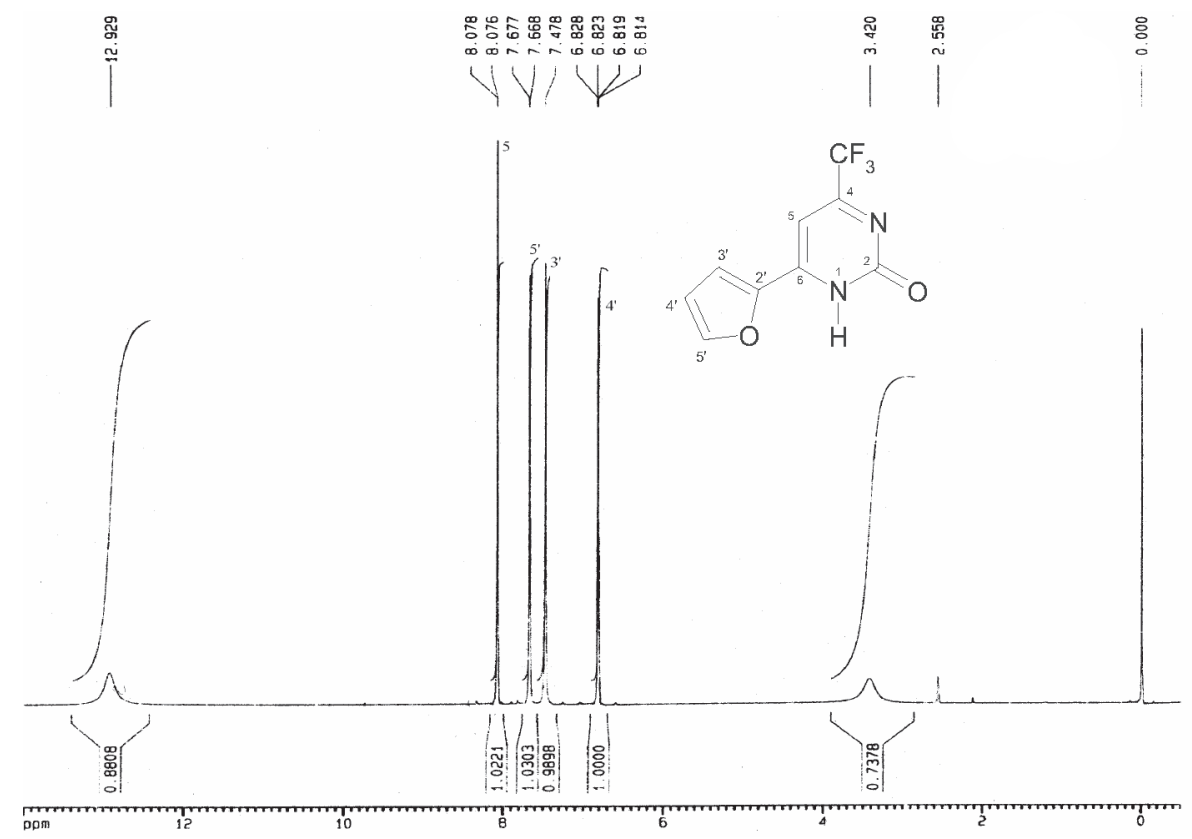

Figure S1. ${ }^{1} \mathrm{H}$ NMR spectrum of the 6-(2-furyl)-4-trifluoromethyl-1 $H$-pyrimidin-2-one (2a), DMSO- $d_{6}$. 


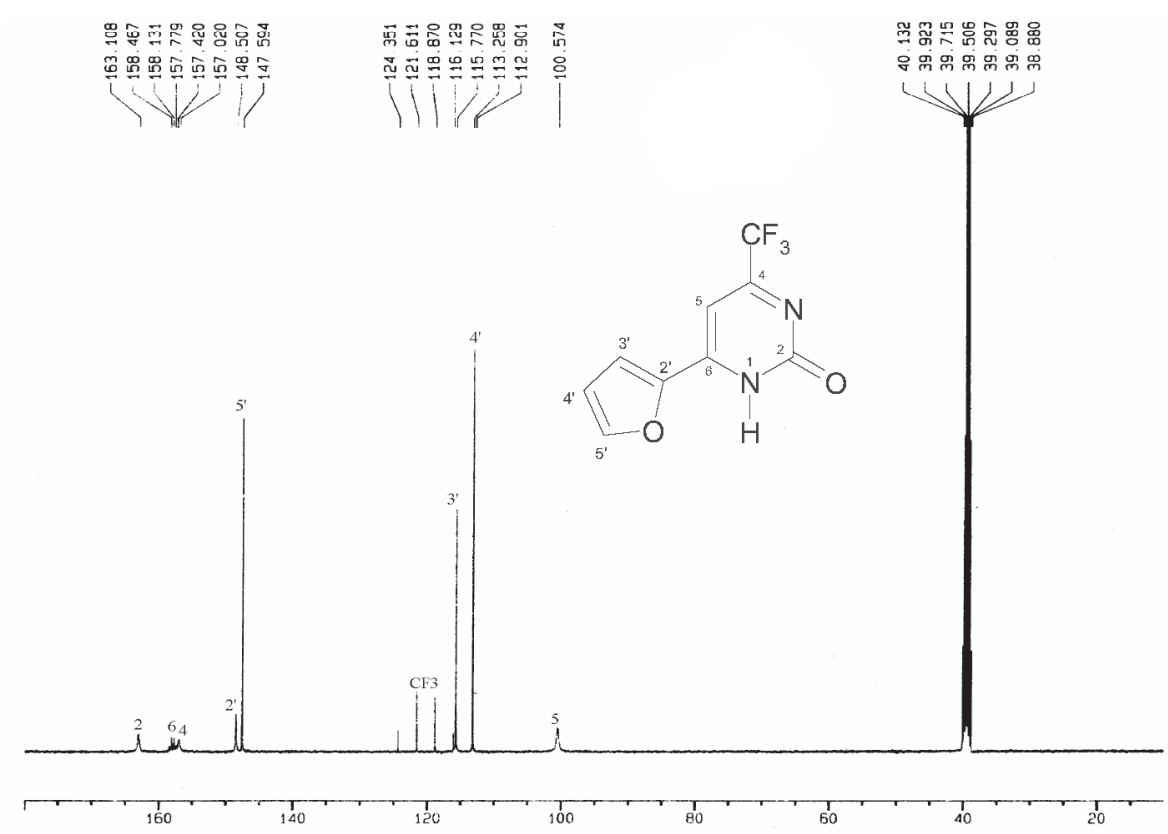

Figure S2. ${ }^{13} \mathrm{C}[\mathrm{H}]$ NMR spectrum of the 6-(2-furyl)-4-trifluoromethyl-1H-pyrimidin-2-one (2a), DMSO- $d_{6}$.
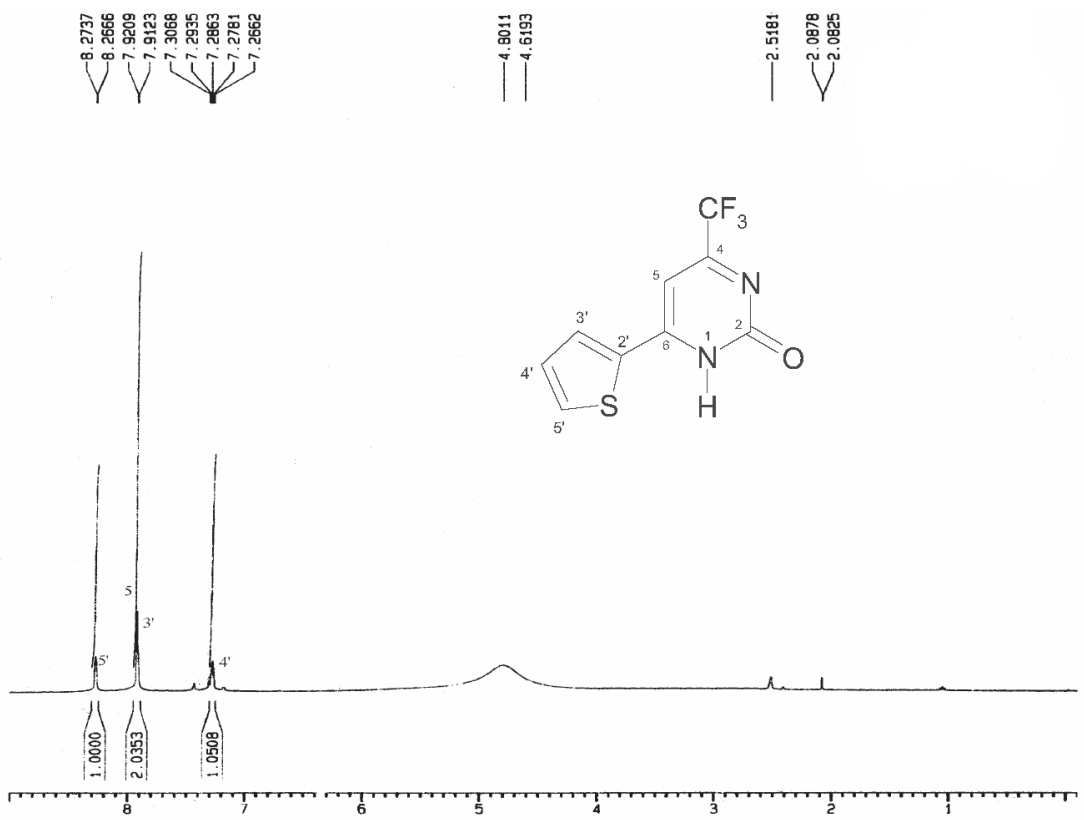

Figure S3. ${ }^{1} \mathrm{H}$ NMR spectrum of the 6-(2-thienyl)-4-trifluoromethyl-1 $H$-pyrimidin-2-one (2b), DMSO- $d_{6}$. 


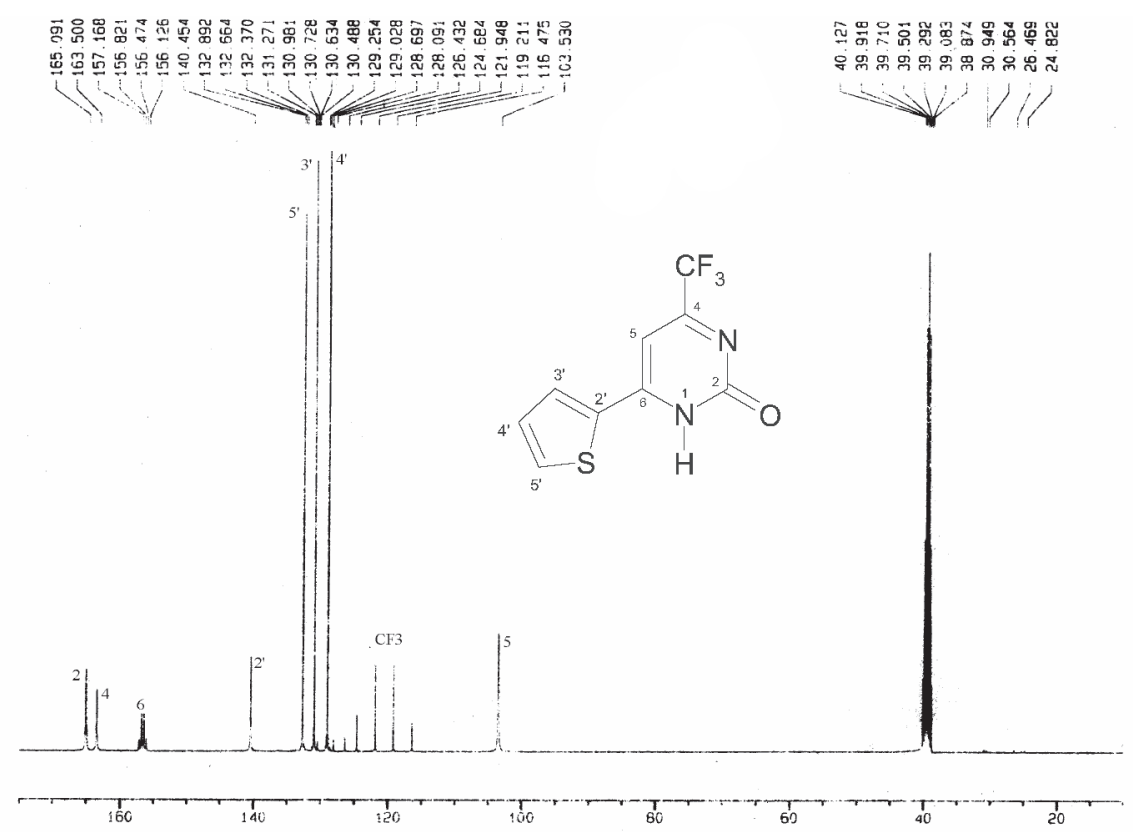

Figure S4. ${ }^{13} \mathrm{C}$ NMR spectrum of the 6-(2-thienyl)-4-trifluoromethyl-1 $H$-pyrimidin-2-one (2b), DMSO- $d_{6}$.

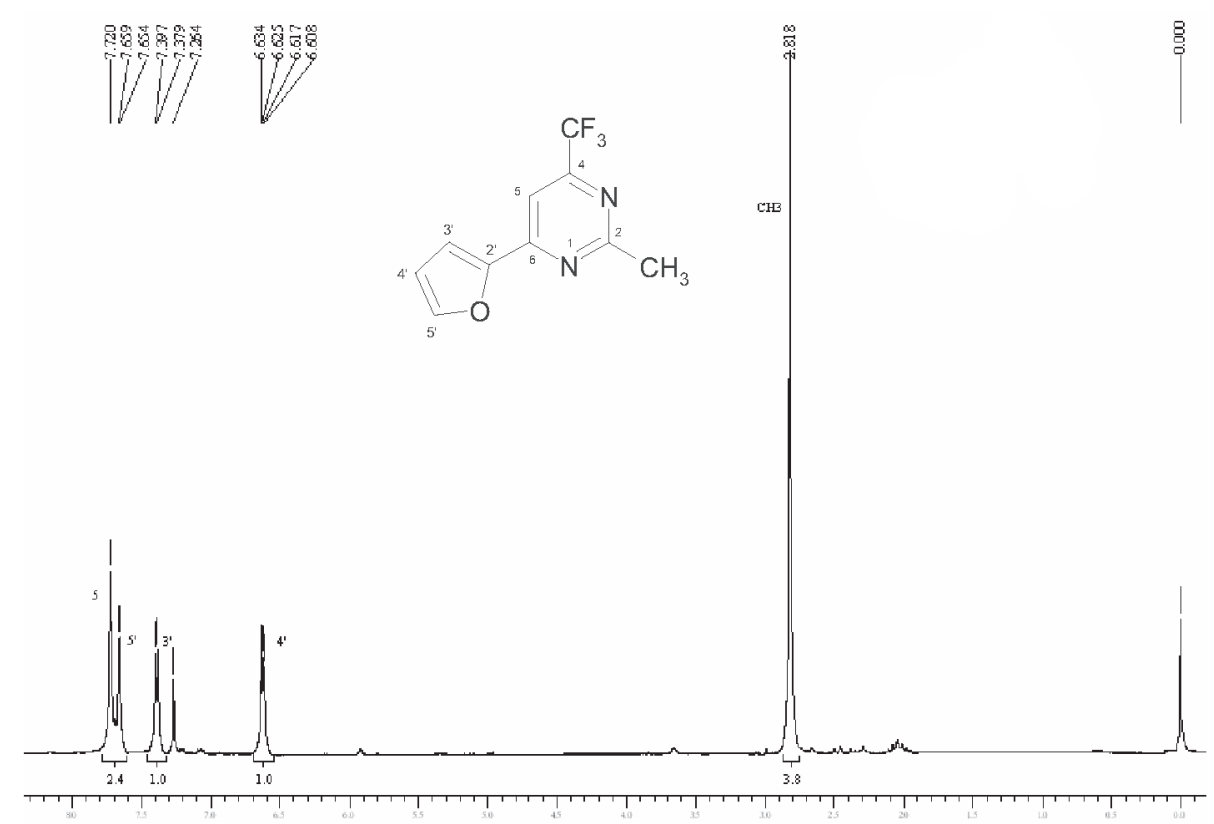

Figura S5. ${ }^{1} \mathrm{H}$ NMR spectrum of the 6-(2-furyl)-2-methyl-4-trifluoromethylpyrimidine (3a), $\mathrm{CDCl}_{3}$. 

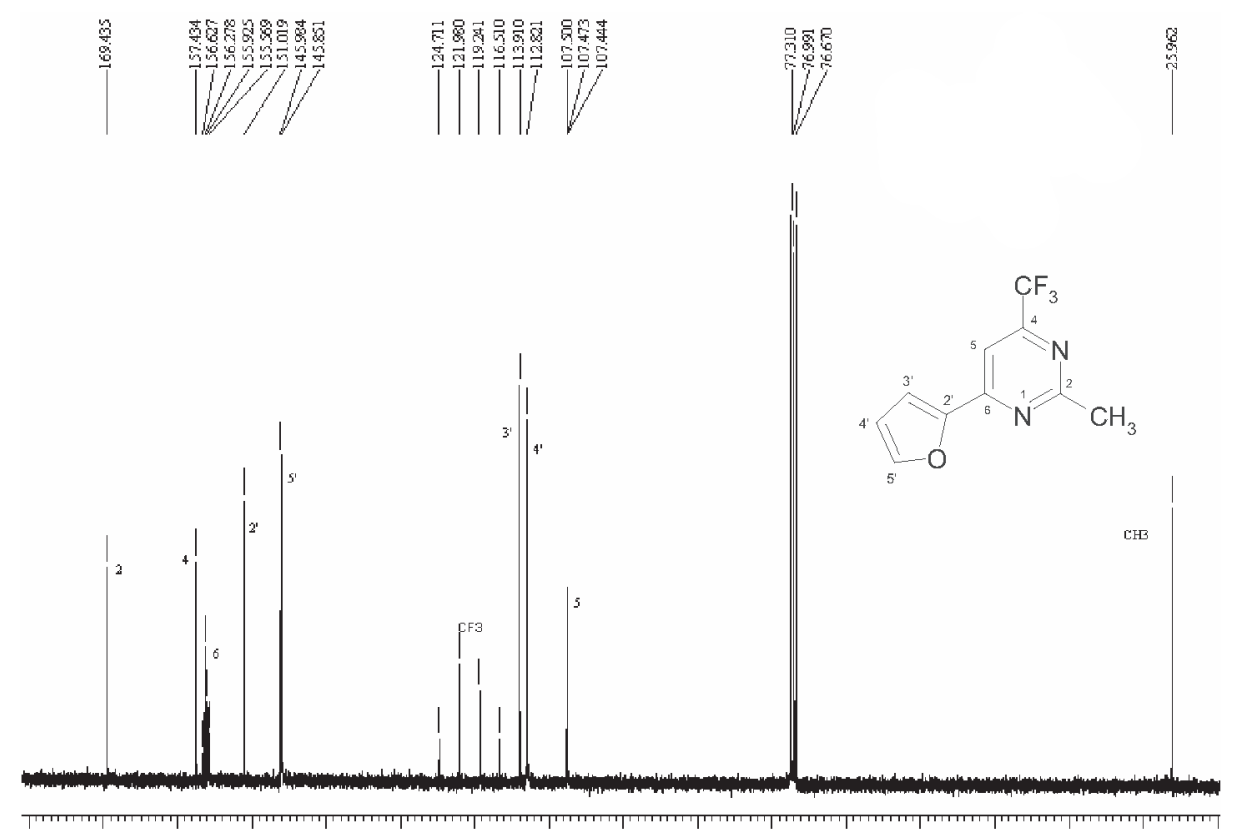

Figure S6. ${ }^{13} \mathrm{C}$ NMR spectrum of the 6-(2-furyl)-2-methyl-4-trifluoromethylpyrimidine (3a), $\mathrm{CDCl}_{3}$.

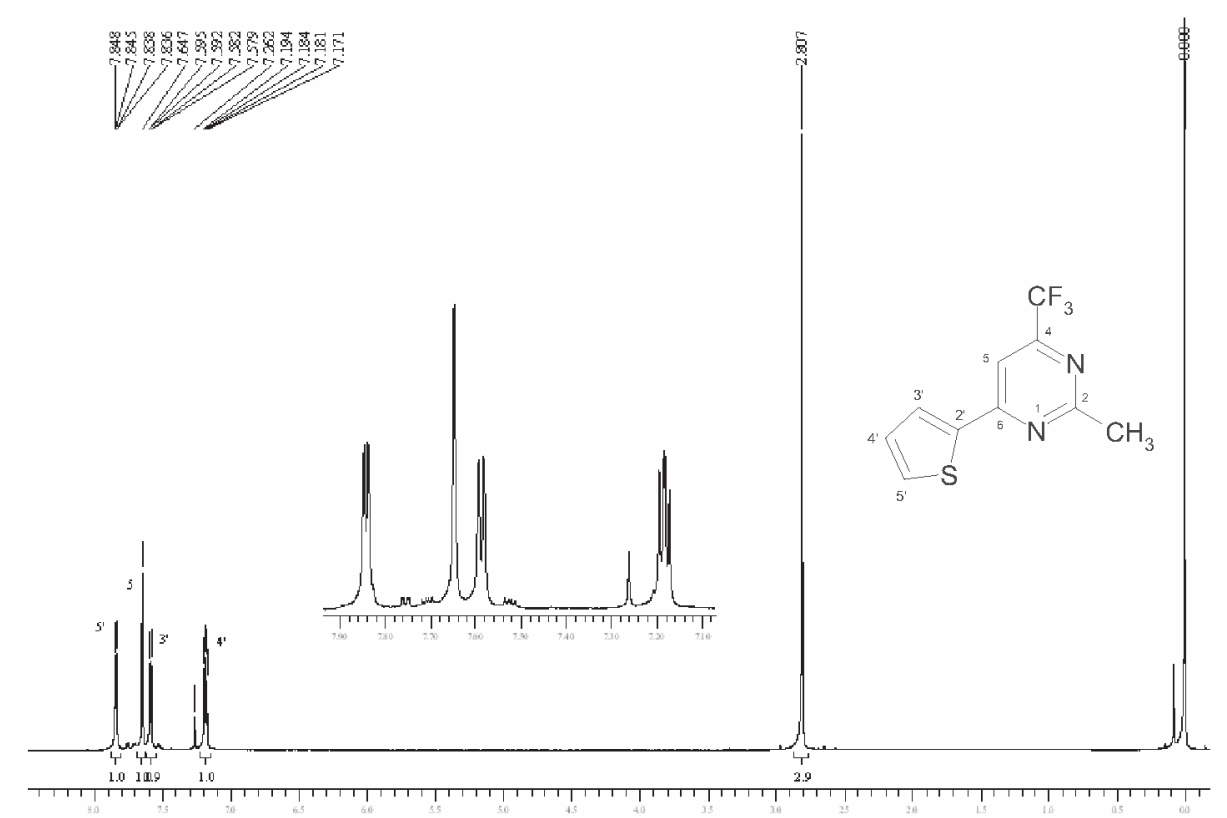

Figure S7. 'H NMR spectrum of the 2-methyl-6-(2-thienyl)-4-trifluoromethylpyrimidine (3b), $\mathrm{CDCl}_{3}$. 

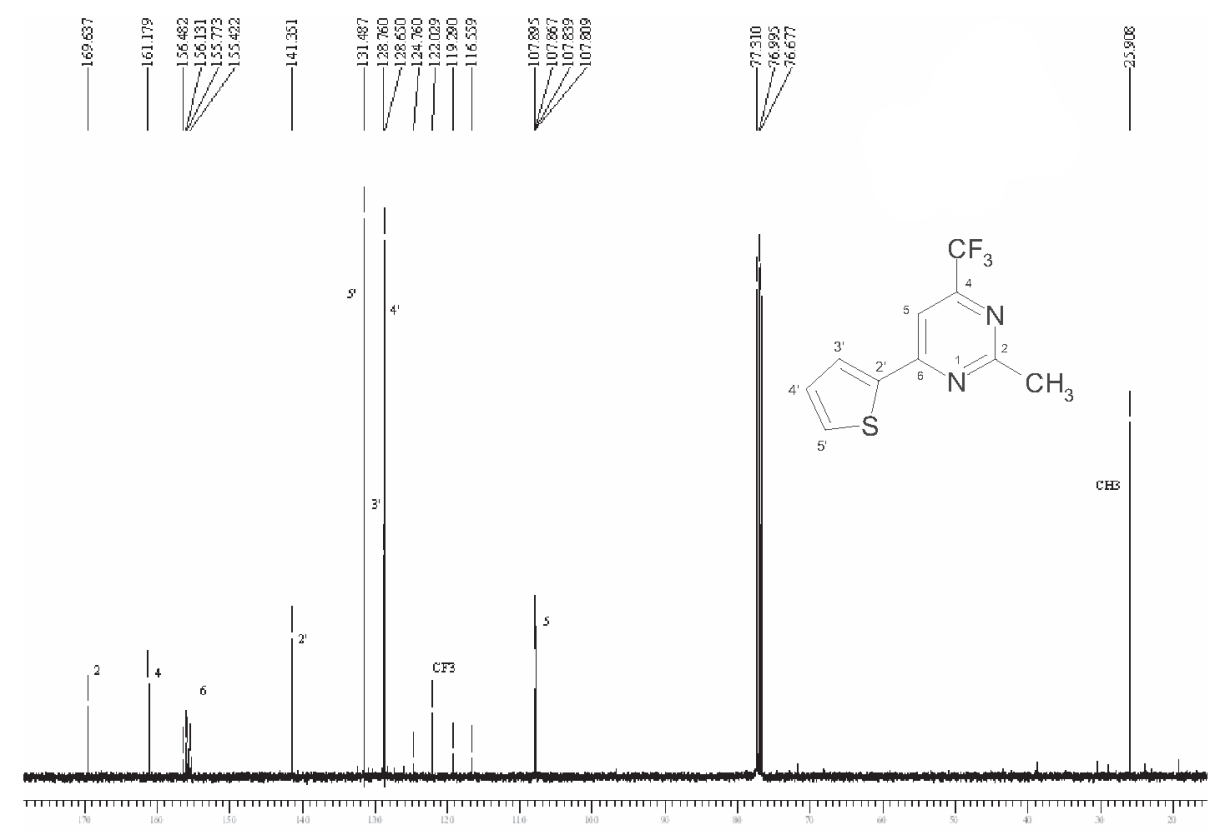

Figure S8. ${ }^{13} \mathrm{C}[\mathrm{H}]$ NMR spectrum of the 6-(2-thienyl)-2-methyl-4-trifluoromethylpyrimidine $\mathbf{3 b}, \mathrm{CDCl}_{3}$.

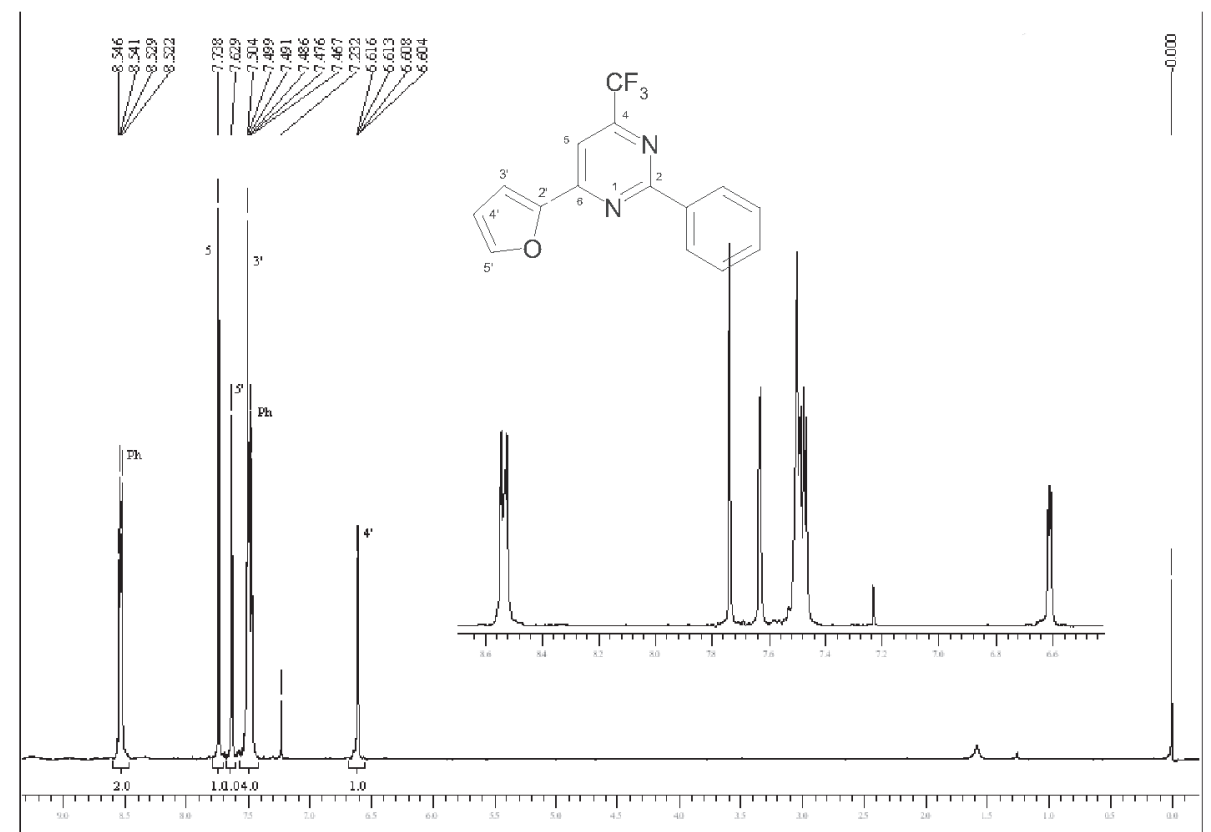

Figure S9. ${ }^{~} \mathrm{H}$ NMR spectrum of the 6-(2-furyl)- 2-phenyl-4-trifluoromethylpyrimidine $4 \mathrm{a}, \mathrm{CDCl}_{3}$. 

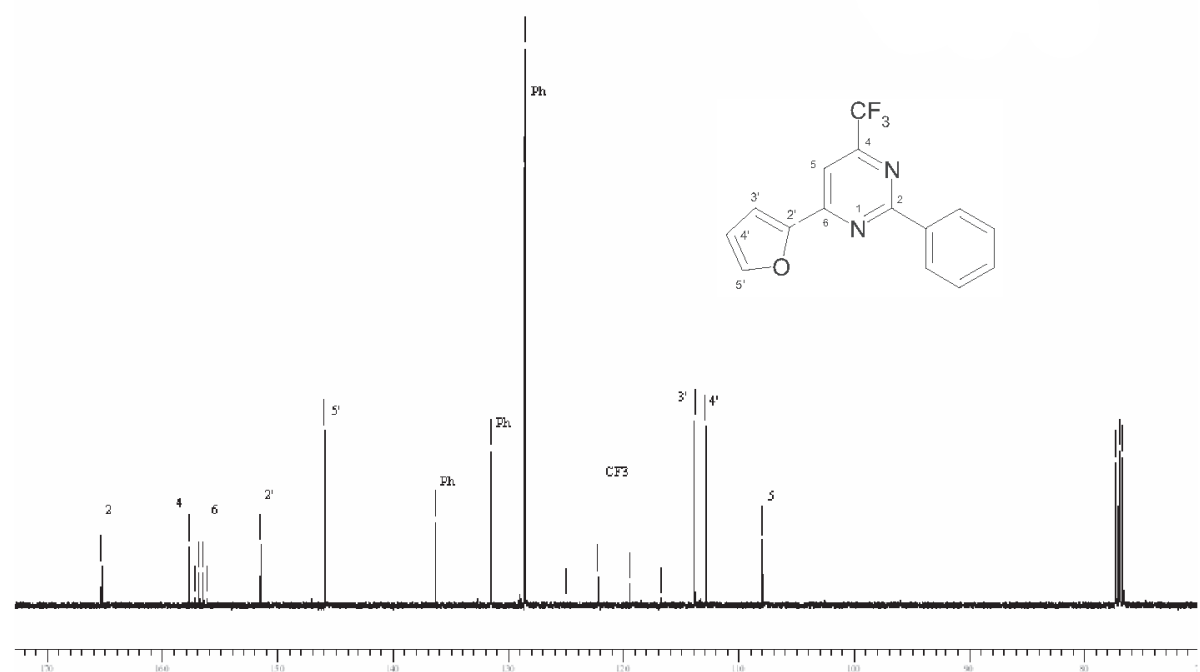

Figure S10. ${ }^{13} \mathrm{C}$ NMR spectrum of the 6-(2-furyl)-2-phenyl-4-trifluoromethylpyrimidine $4 a, \mathrm{CDCl}_{3}$.

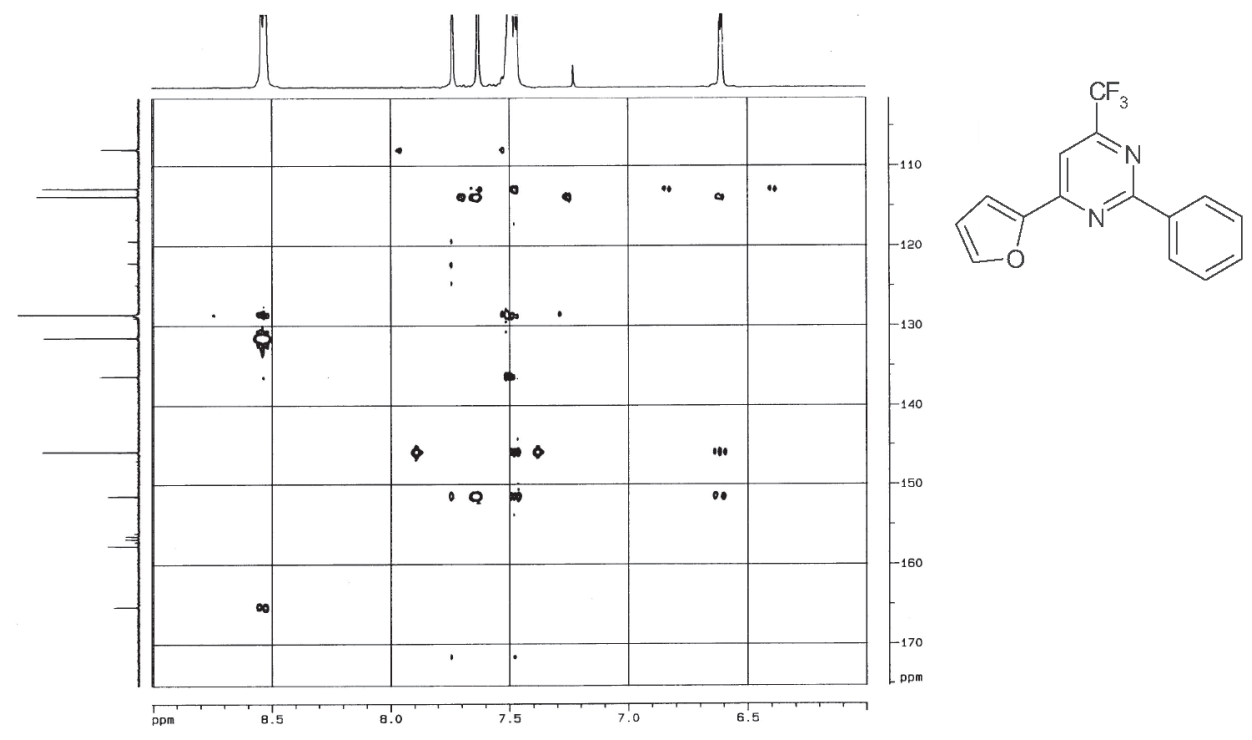

Figure S11. HMBC spectrum of the 6-(2-furyl)-2-phenyl-4-trifluoromethylpyrimidine $\mathbf{3 b}, \mathrm{CDCl}_{3}$. 


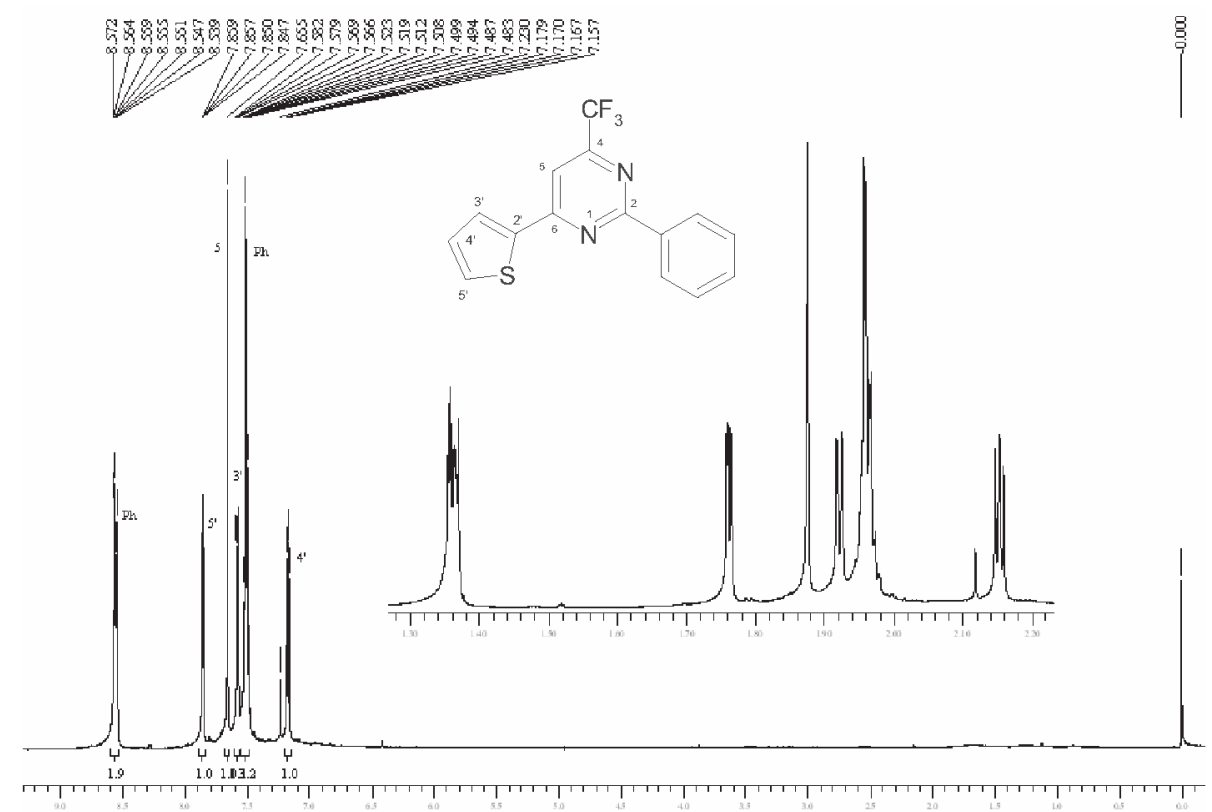

Figure S12. 'H NMR spectrum of the 2-phenyl-6-(2-thienyl)-4-trifluoromethylpyrimidine $4 \mathbf{b}, \mathrm{CDCl}_{3}$.

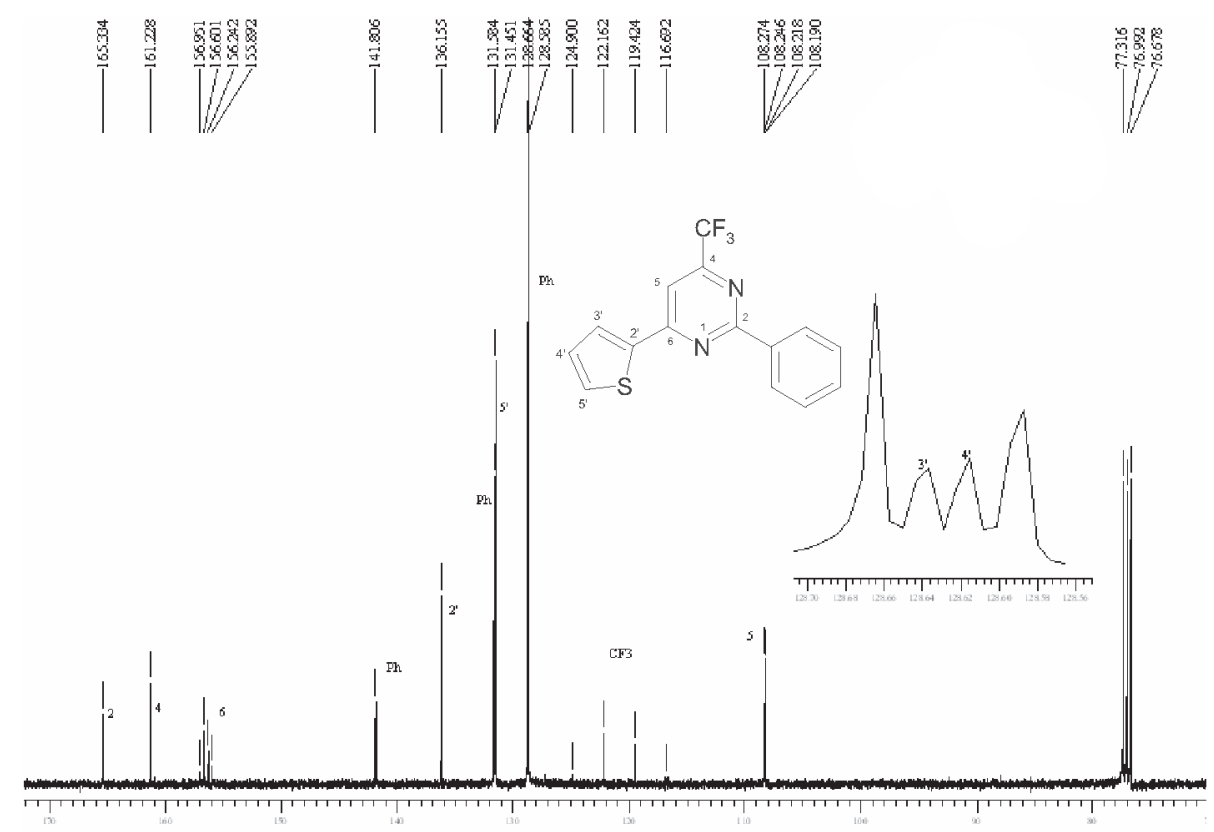

Figure S13. ${ }^{13} \mathrm{C}[\mathrm{H}]$ NMR spectrum of the 2-phenyl-6-(2-thienyl)-4-trifluoromethylpyrimidine $\mathbf{4 b}, \mathrm{CDCl}_{3}$. 


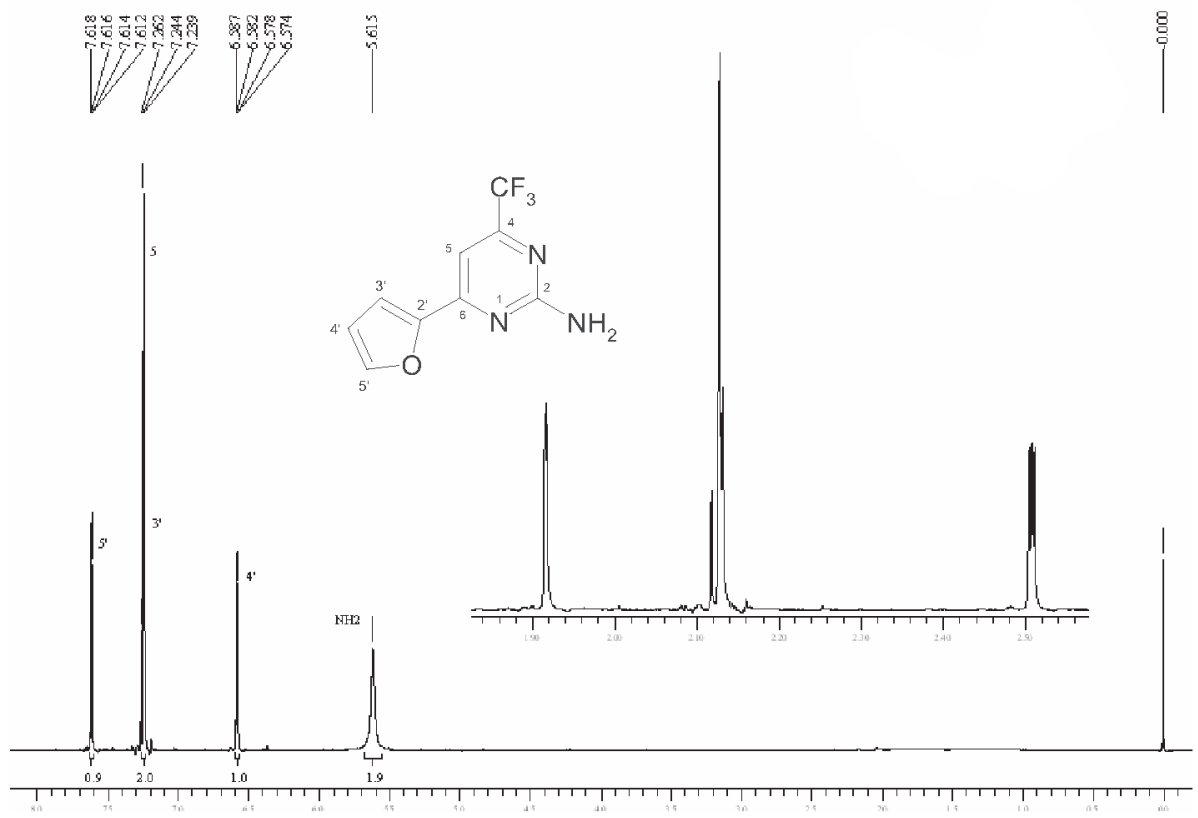

Figure S14. ${ }^{1} \mathrm{H}$ NMR spectrum of the 2-amino-6-(2-furyl)-4-trifluoromethylpyrimidine $\mathbf{5 a}, \mathrm{CDCl}_{3}$.
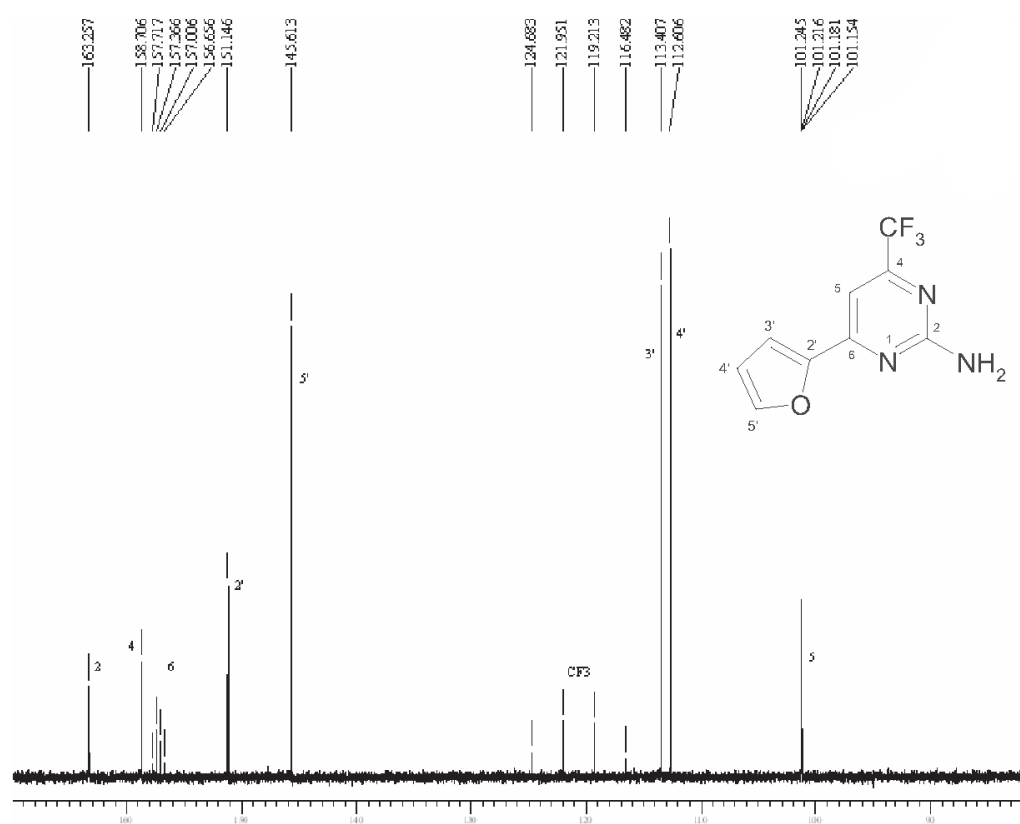

Figure S15. ${ }^{13} \mathrm{C}[\mathrm{H}] \mathrm{NMR}$ spectrum of the 2-amino-6-(2-furyl)-4-trifluoromethylpyrimidine 5a, $\mathrm{CDCl}_{3}$. 


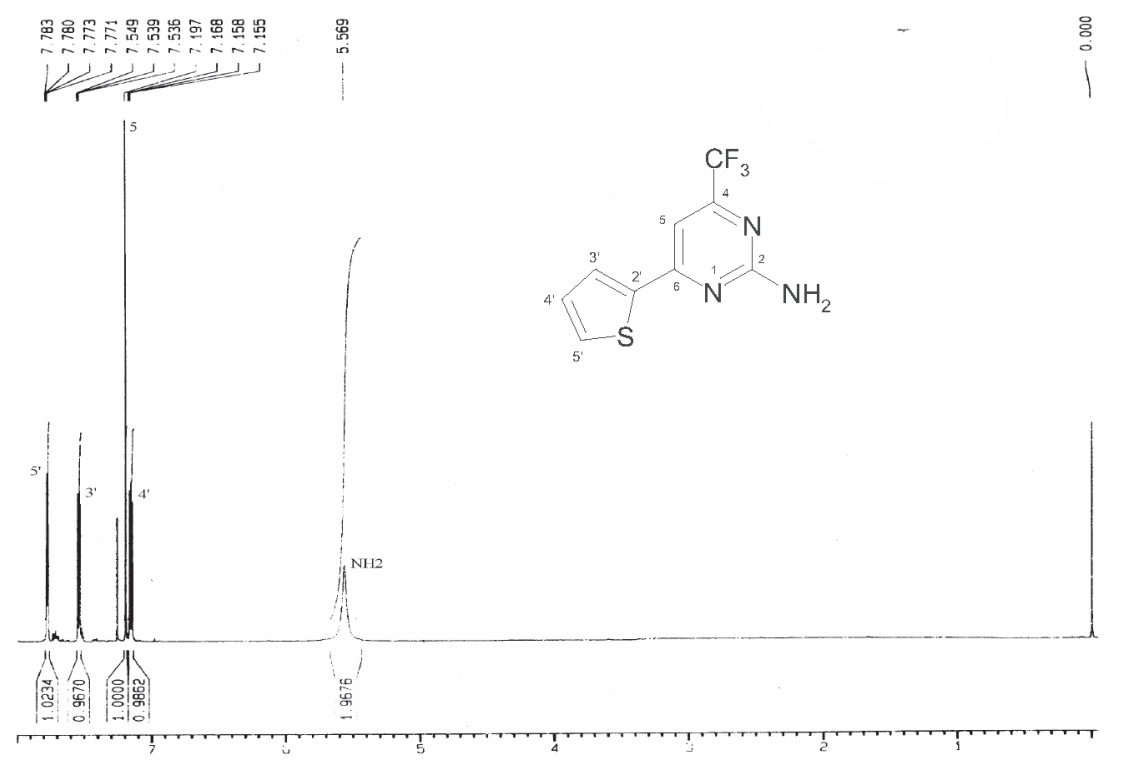

Figure S16. ${ }^{1} \mathrm{H}$ NMR spectrum of the 2-amino-6-(2-thienyl)-4-trifluoromethylpyrimidine $\mathbf{5 b}, \mathrm{CDCl}_{3}$.

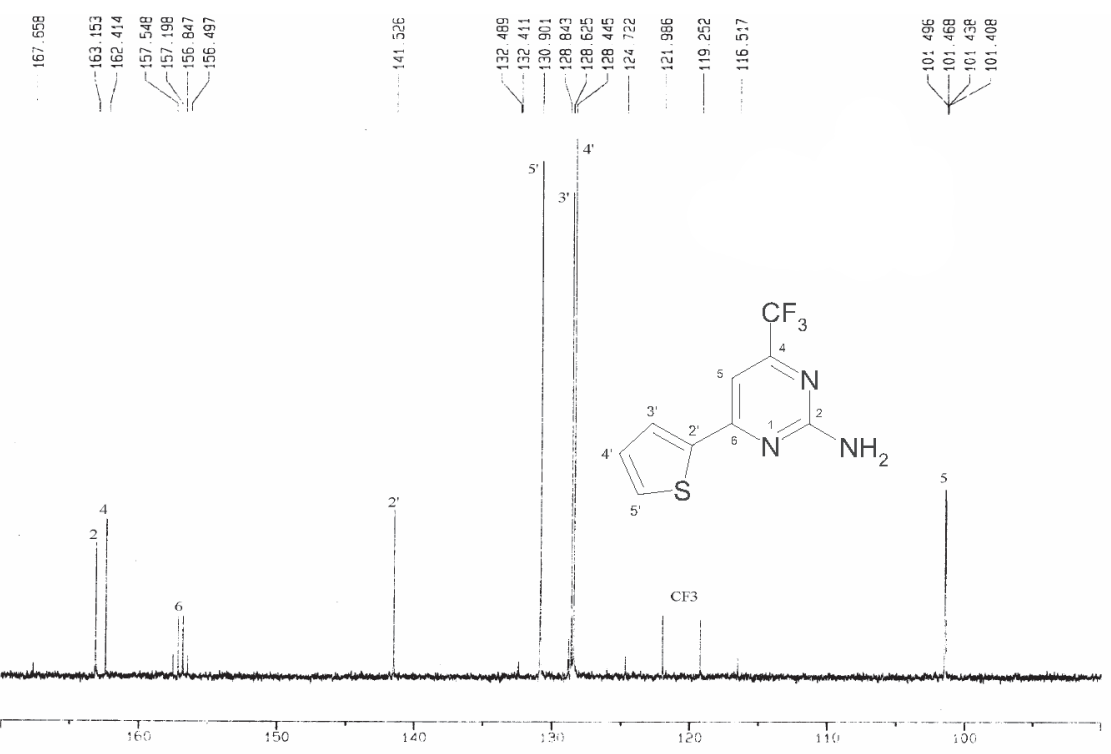

Figure S17. ${ }^{13} \mathrm{C}[\mathrm{H}]$ NMR spectrum of the 2-amino-6-(2-thienyl)-4-trifluoromethylpyrimidine $\mathbf{5 b}, \mathrm{CDCl}_{3}$. 


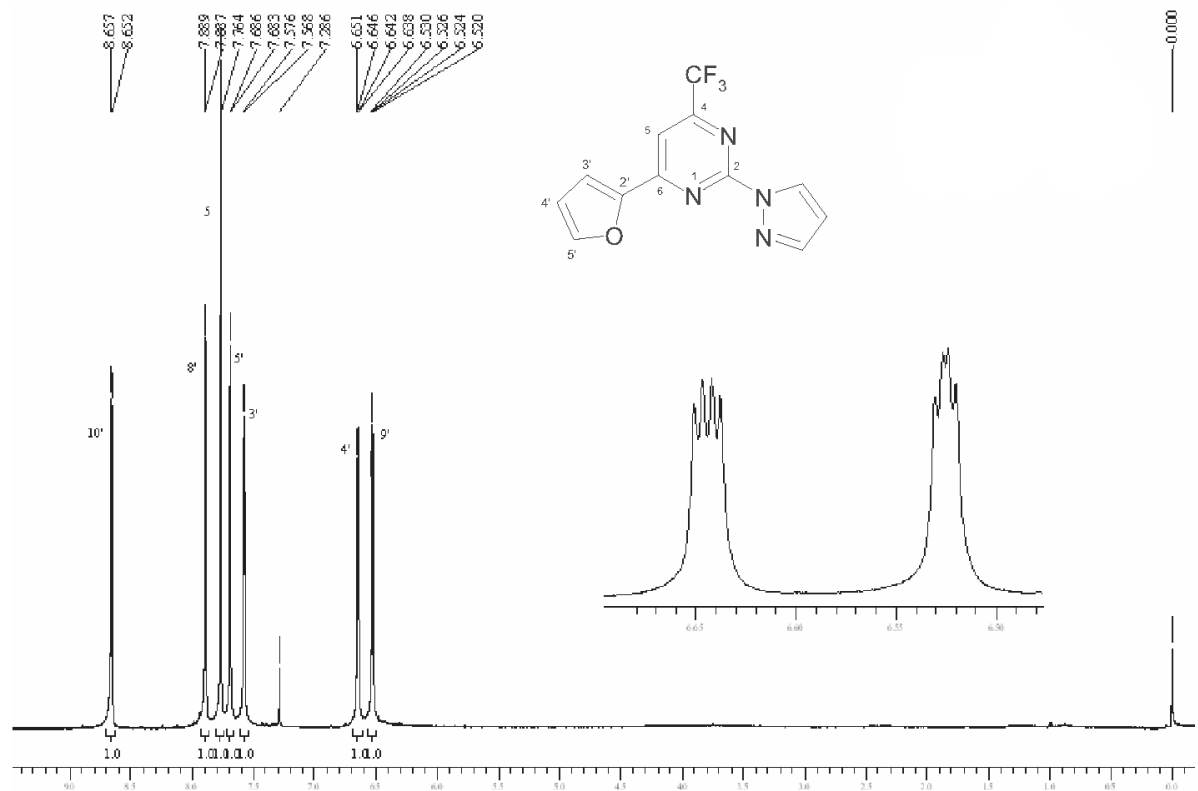

Figure S18. ${ }^{1} \mathrm{H}$ NMR spectrum of the 6-(2-furyl)-2-(1H-pyrazol-1-yl)-4-trifluoromethyl-pyrimidine 6 a, $\mathrm{CDCl}_{3}$.

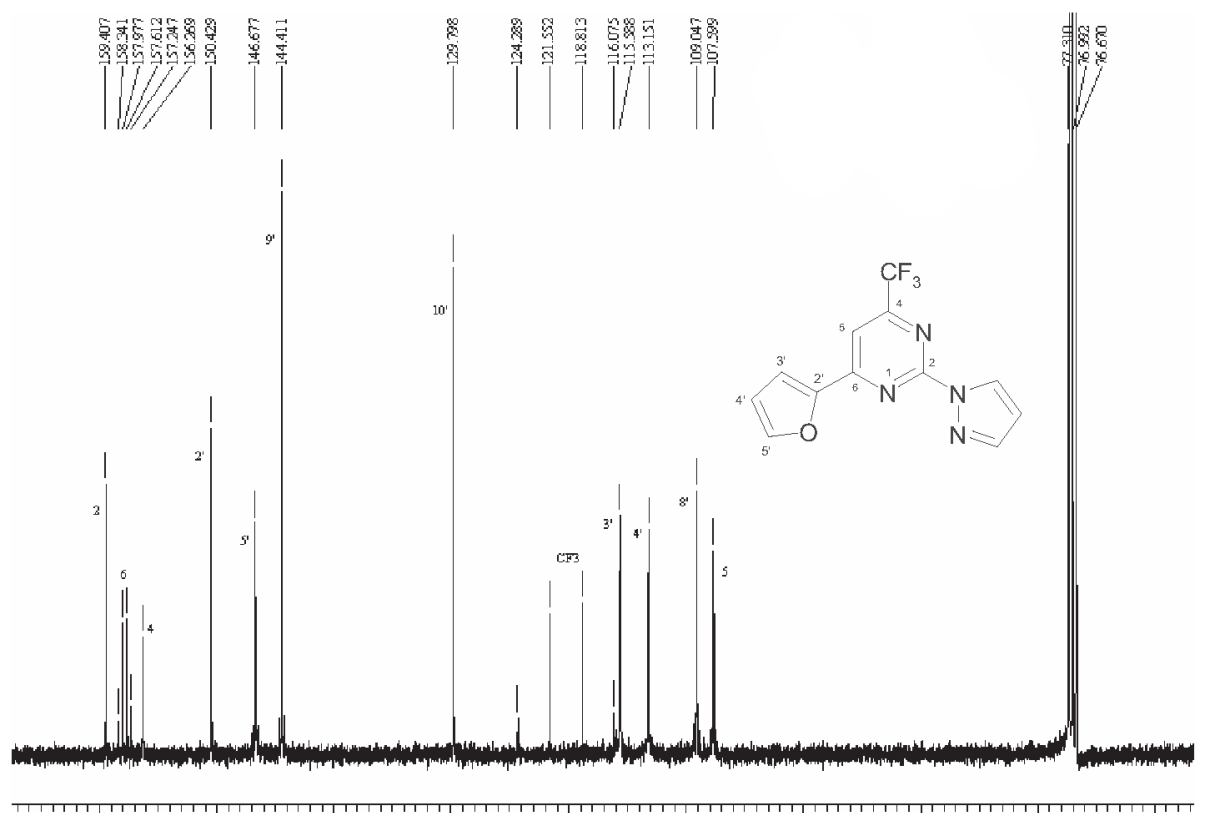

Figure S19. ${ }^{13} \mathrm{C}[\mathrm{H}]$ NMR spectrum of 6-(2-furyl)-2-(1H-pyrazol-1-yl)-4-trifluoromethyl-pyrimidine $\mathbf{6 a}, \mathrm{CDCl}_{3}$. 


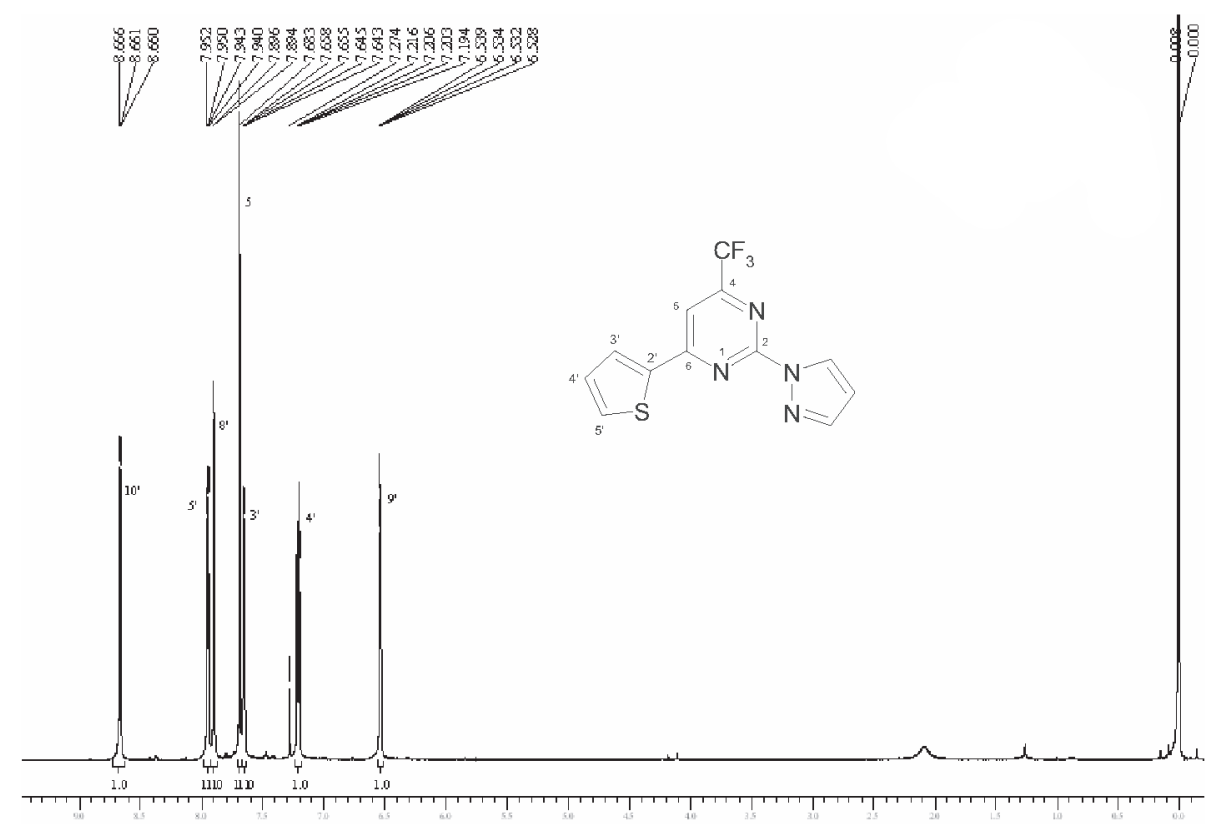

Figure S20. ${ }^{1} \mathrm{H}$ NMR spectrum of the 2-(1H-pyrazol-1-yl)-6-(2-thienyl) 4-trifluoromethylpyrimidine $\mathbf{6 b}, \mathrm{CDCl}_{3}$.
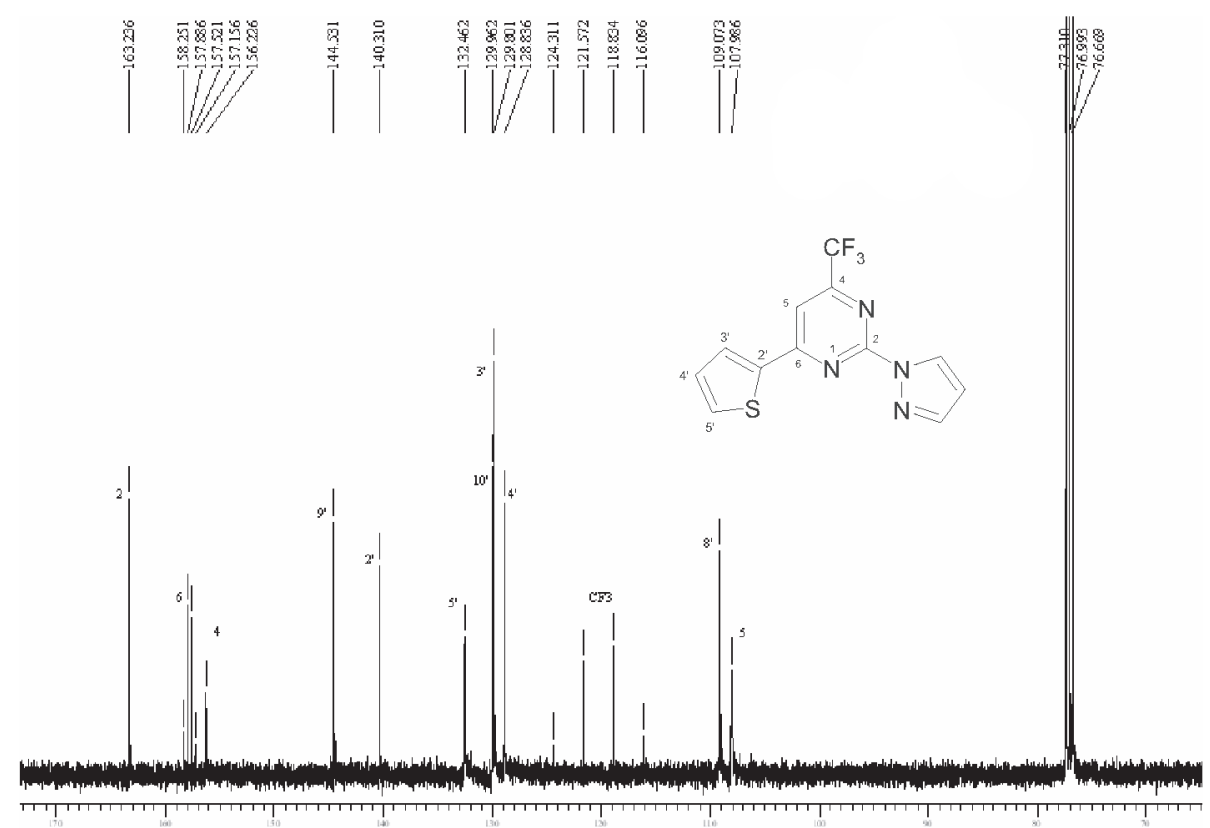

Figure S21. ${ }^{13} \mathrm{C}[\mathrm{H}]$ NMR spectrum of the 2-(1H-pyrazol-1-yl)-6-(2-thienyl)-4-trifluoromethylpyrimidine $\mathbf{6 b}, \mathrm{CDCl}_{3}$. 

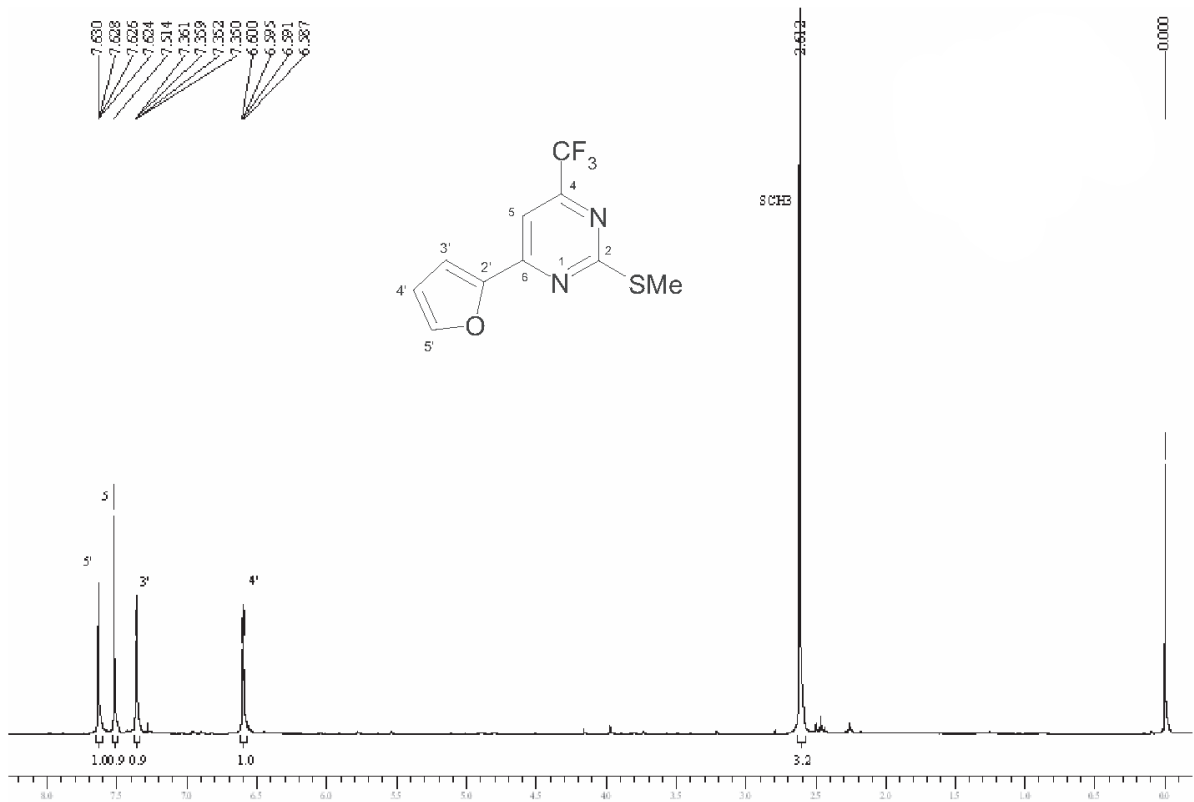

Figure S22. ${ }^{1} \mathrm{H}$ NMR spectrum of the 6-(2-furyl)-2-tiomethyl-4-trifluoromethylpyrimidine 7a, $\mathrm{CDCl}_{3}$.

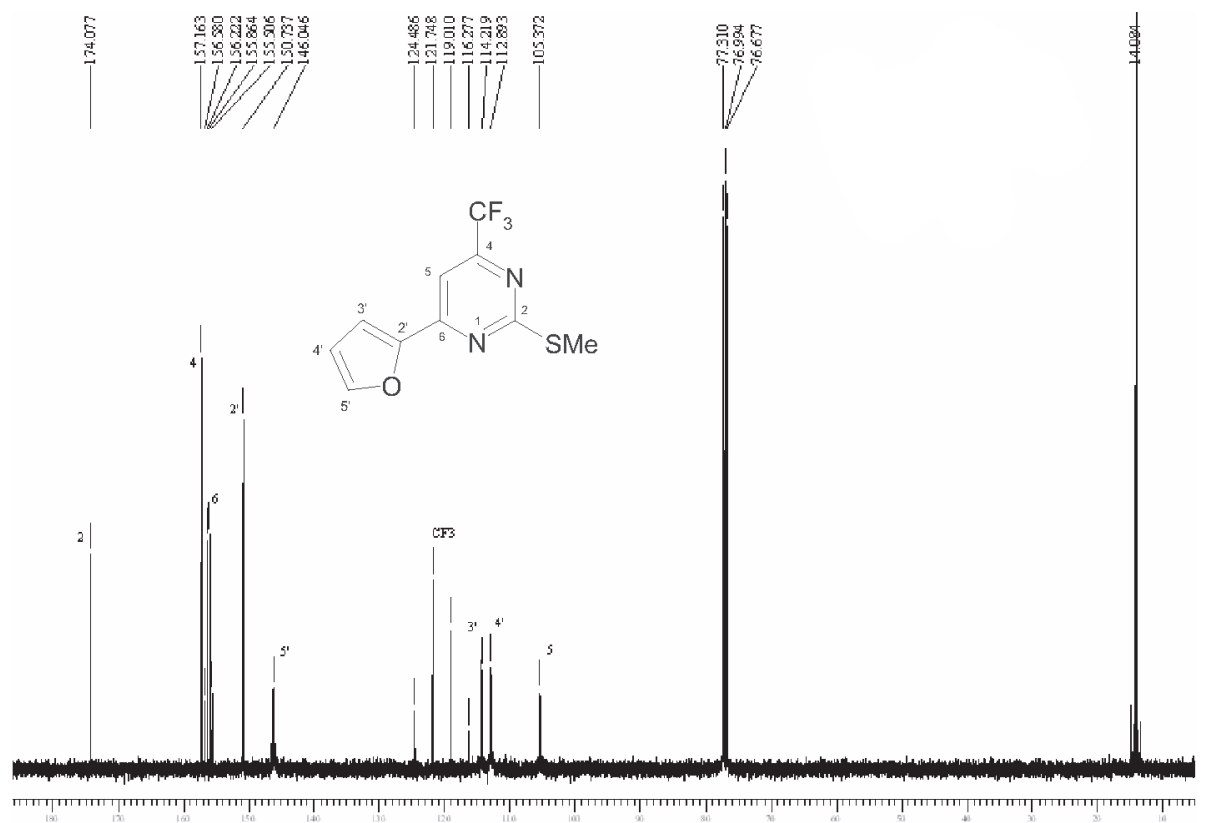

Figure S23. ${ }^{13} \mathrm{C}[\mathrm{H}]$ NMR spectrum of the 6-(2-furyl)-2-tiomethyl-4-trifluoromethylpyrimidine $7 \mathbf{a}, \mathrm{CDCl}_{3}$. 


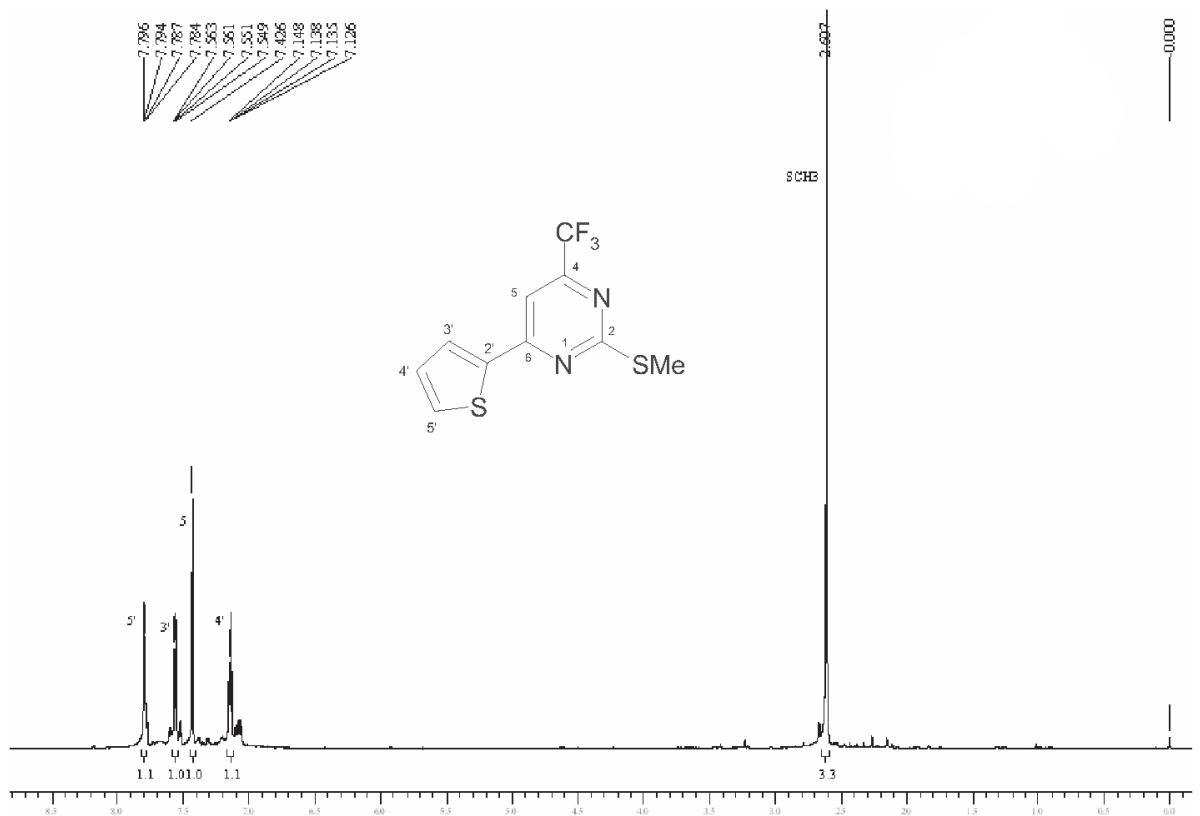

Figure S24. ${ }^{1} \mathrm{H}$ NMR spectrum of the 6-(2-thienyl)-2-tiomethyl-4-trifluoromethylpyrimidine $7 \mathbf{b}, \mathrm{CDCl}_{3}$.

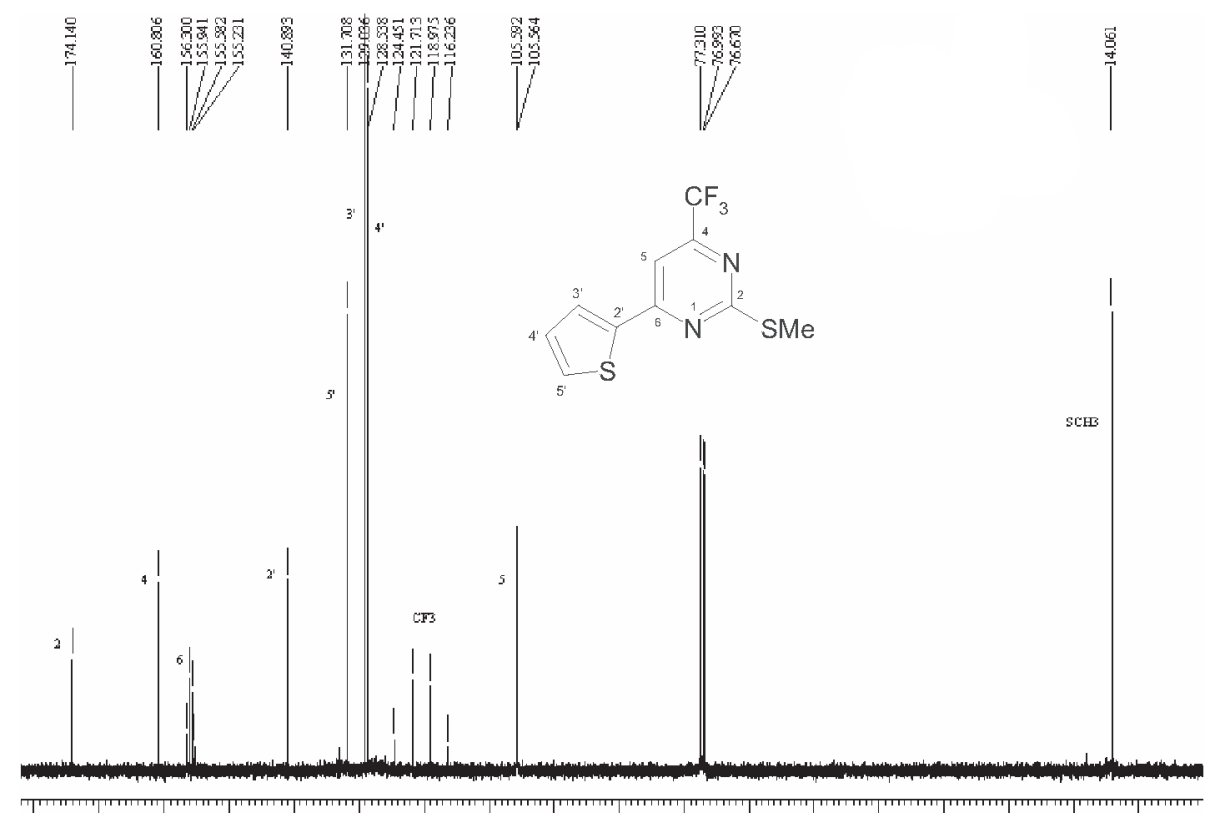

Figure S25. ${ }^{13} \mathrm{C}[\mathrm{H}]$ NMR spectrum of the 6-(2-thienyl)-2-tiomethyl-4-trifluoromethylpyrimidine $\mathbf{7 b}, \mathrm{CDCl}_{3}$. 


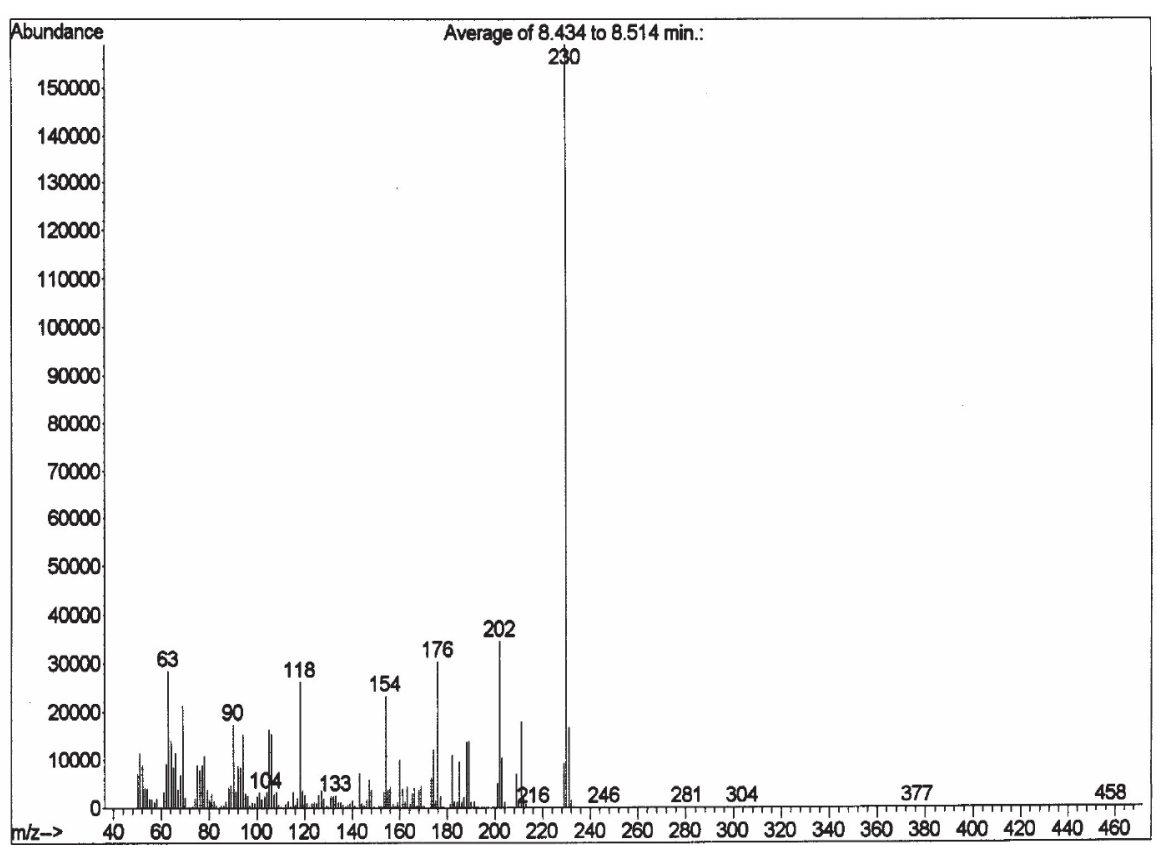

Figure S26. MS of 6-(2-furyl)-4-trifluoromethyl-1H-pyrimidin-2-one (2a).

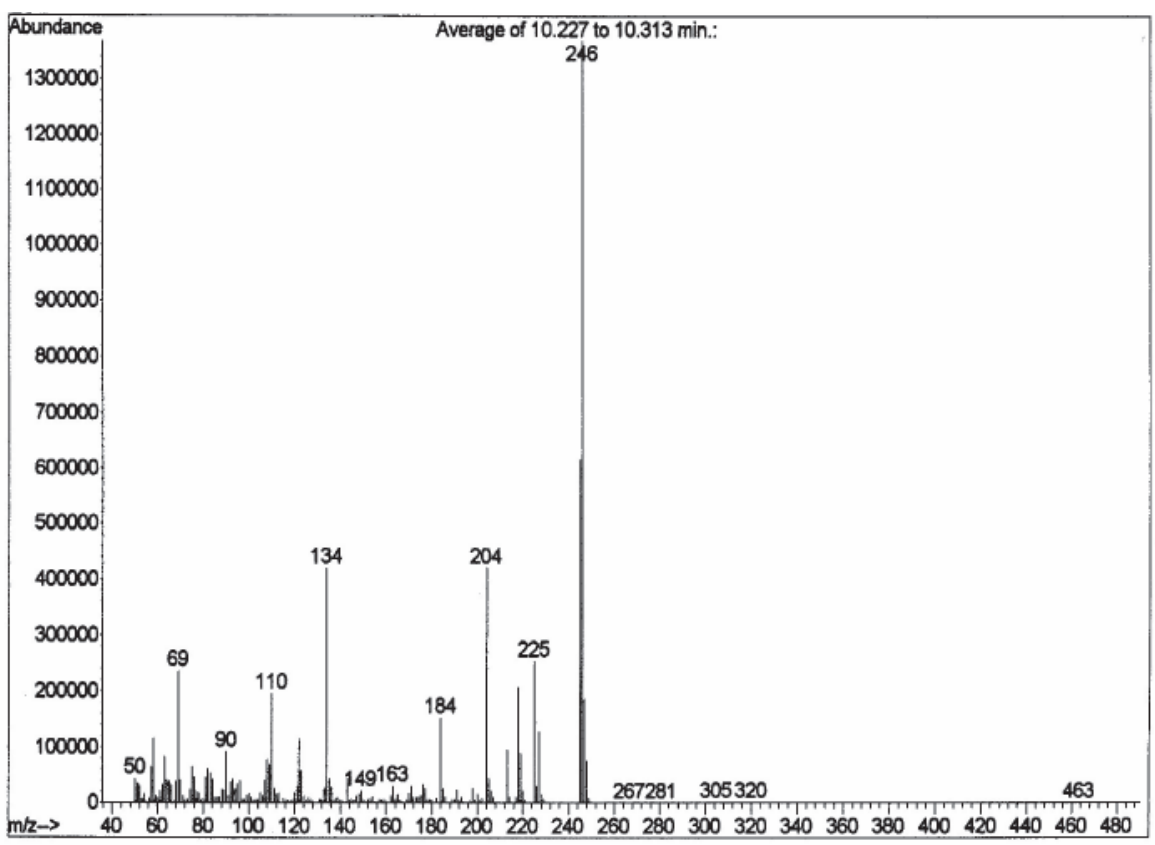

Figure S27. MS of 6-(2-thienyl)-4-trifluoromethyl-1H-pyrimidin-2-one (2b). 


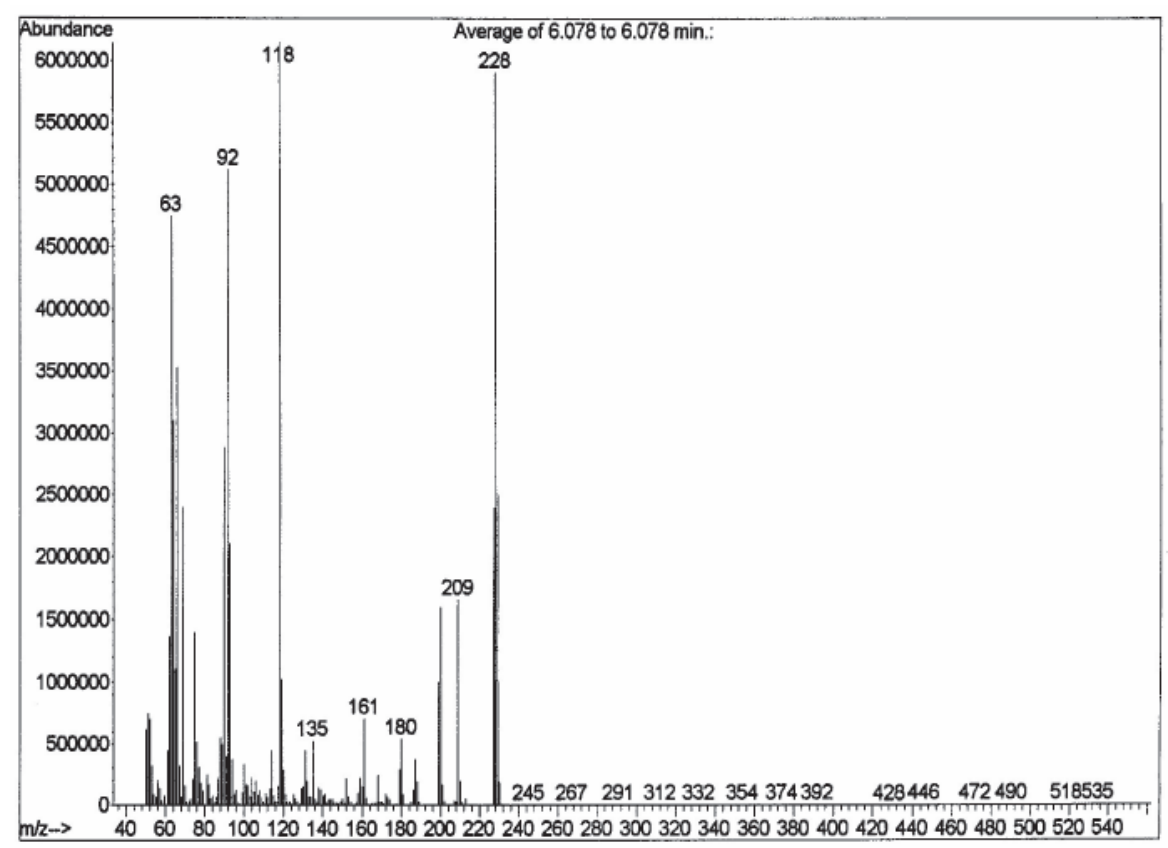

Figure S28. MS of 6-(2-furyl)-2-methyl-4-trifluoromethylpyrimidine (3a).

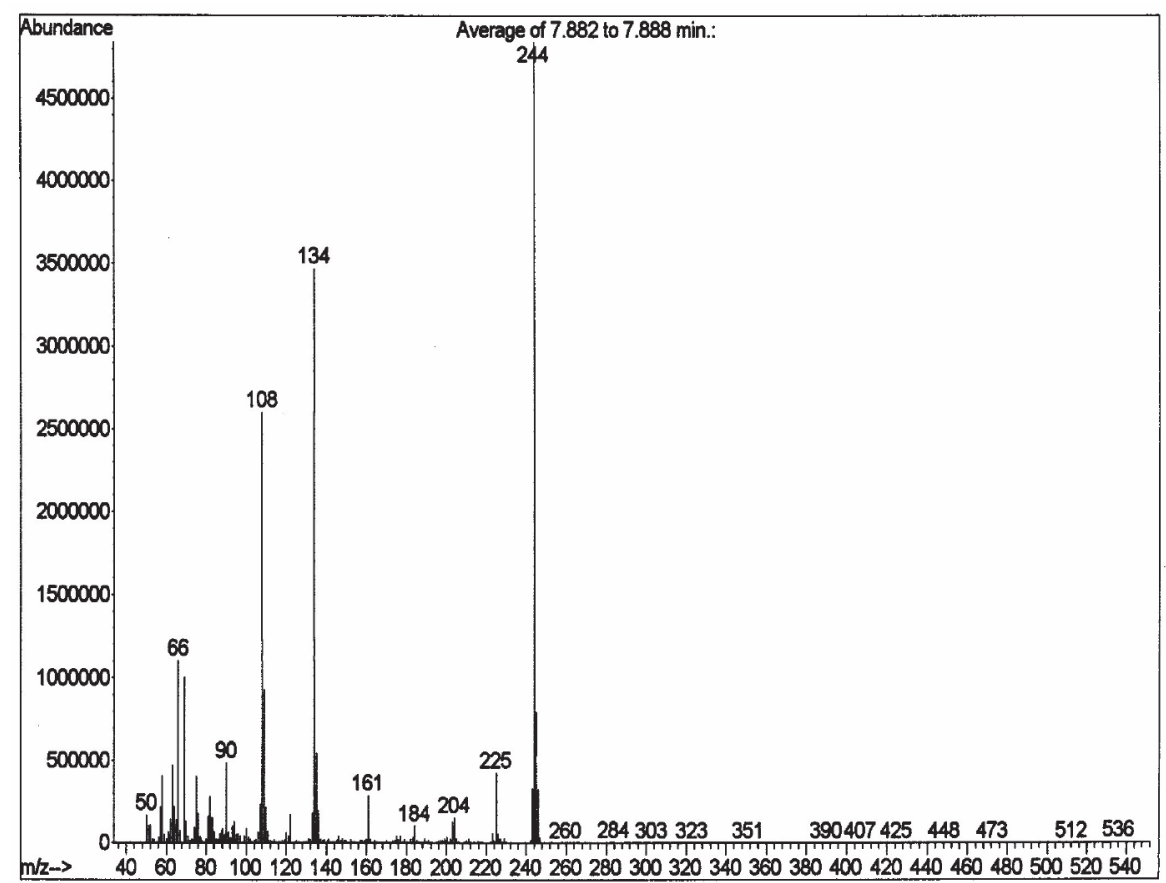

Figure S29. MS of 2-methyl-6-(2-thienyl)-4-trifluoromethylpyrimidine (3b). 


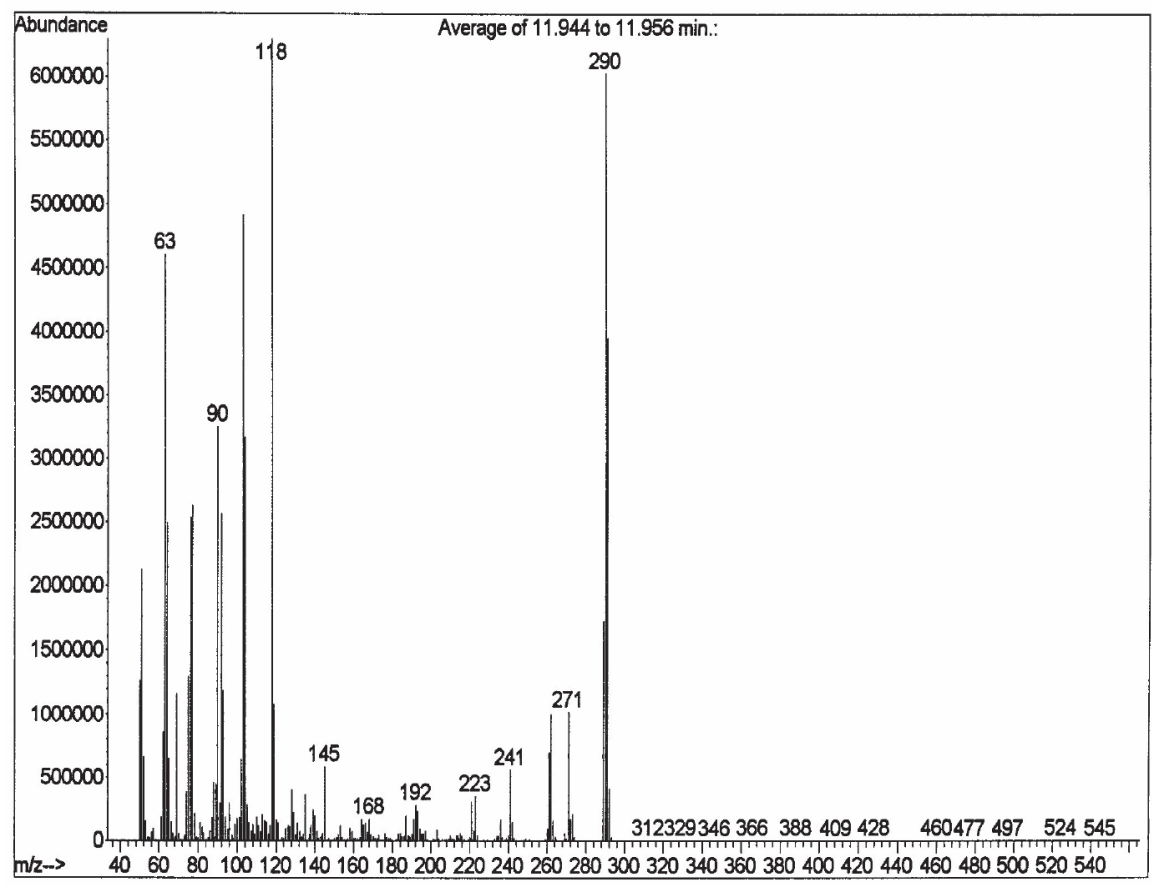

Figure S30. MS of 6-(2-furyl)- 2-phenyl-4-trifluoromethyl-pyrimidine (4a).

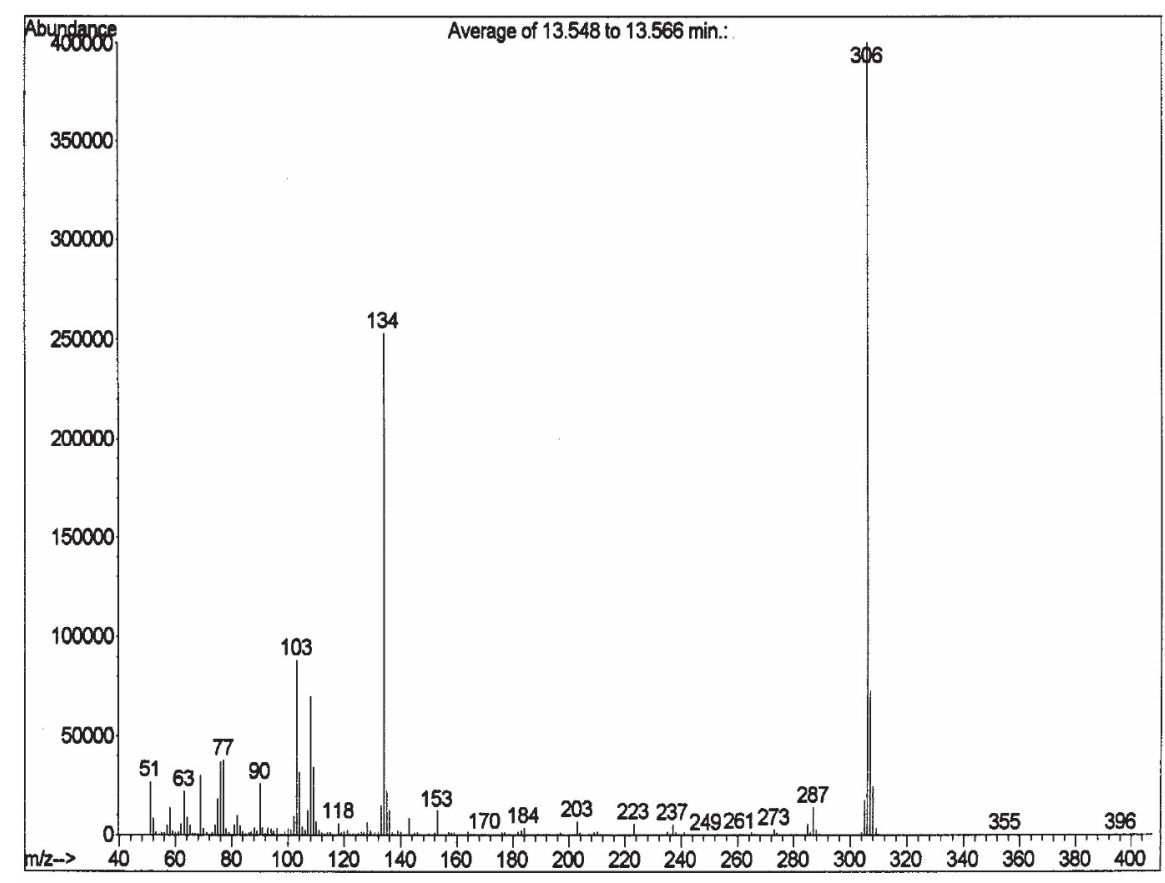

Figure S31. MS of 2-phenyl-6-(2-thienyl)-4-trifluoromethylpyrimidine (4b). 